\title{
Joint User Scheduling, Precoder Design and Transmit Direction Selection in MIMO TDD Small Cell Networks
}

\author{
Sandra Lagen, Adrian Agustin, Member, IEEE, and Josep Vidal, Member, IEEE
}

\begin{abstract}
New short-length single-direction frame structures are proposed for 5G time division duplex (TDD) systems, where the transmit direction (i.e. either downlink (DL) or uplink (UL)) can be independently chosen at each cell in every frame. Accordingly, high flexibility is provided to match the per-cell DL/UL traffic asymmetries and full exploitation of dynamic TDD is allowed. As a downside, interference management becomes crucial. In this regard, this paper proposes a procedure for dynamic TDD in dense MIMO small cell networks, where the transmit direction selected per small cell (SC) is dynamically optimized together with the user scheduling and transmit precoding. We focus on the maximization of a general utility function that takes into account the DL/UL traffic asymmetries of each user and the interference conditions in the network. Although the problem is non-convex, it is decomposed thanks to the interferencecost concept and then efficiently solved in parallel. Simulation results show gains in DL and $U L$ average rates for different traffic asymmetries and SC/user densities as compared to existing dynamic TDD schemes thanks to the proposed joint optimization. The gains become more significant when there is high interference and limited number of antennas.
\end{abstract}

Keywords-Dynamic TDD, small cell networks, 5G shortlength single-direction frame structure, MIMO, user scheduling, precoder design, power allocation, transmit direction selection.

\section{INTRODUCTION}

Dense multiple-input multiple-output (MIMO) small cell networks (SCNs) are considered a key technology for fifth generation $(5 \mathrm{G})$ systems as a result of their cost-effectiveness in boosting the area spectral efficiency through densification of the network with small cells (SCs) [1]. SCs transmit low power and provide short range coverage, so the expected number of users served per SC is reduced. As a consequence, the amount of downlink (DL) and uplink (UL) traffic per cell can vary over space and time more drastically in SCNs than in conventional macrocell-based networks [2].

In contrast to LTE frequency division duplex (FDD) systems, where the amount of band devoted for DL and UL transmissions is fixed and equally divided, LTE time division duplex (TDD) systems allow for asymmetric DL-UL

Copyright (c) 2017 IEEE. Personal use of this material is permitted. However, permission to use this material for any other purposes must be obtained from the IEEE by sending a request to pubs-permissions@ieee.org. Manuscript received April 5, 2016; revised August 6, October 3, November 25, 2016, January 12, 26, 2017; accepted January 31, 2017. The associate editor coordinating the review of this paper and approving it for publication was L. Dai.

The authors are with the Department of Signal Theory and Communications, Universitat Politcnica de Catalunya, Barcelona 08034, Spain (e-mail: sandra.lagen@upc.edu; adrian.agustin@upc.edu; josep.vidal@upc.edu). allocations by providing 7 different semi-statically configured UL-DL configurations of an LTE frame that is composed of 10 LTE subframes [3]. The predefined UL-DL configurations differ in the switching points between a DL and an UL transmission within an LTE frame, hence providing DL-UL allocation ratios that vary from 4:6 to 9:1 (number of DL:UL LTE subframes) [2]. Usually, the UL-DL configuration in LTE TDD is the same for all cells and is determined at the network level based on long-term traffic statistics, which might not match the per-cell traffic asymmetries.

In this regard, the new emerging dynamic TDD technique [4], [5] offers the possibility of a dynamic UL-DL reconfiguration so as to adapt the DL-UL allocation ratio to the traffic asymmetry at each cell. This higher flexibility is specially suited for dense MIMO SCNs because of the asymmetric traffic conditions. As a downside, new types of interference appear in the system (i.e. DL-to-UL and UL-to-DL interference). So, advanced interference management procedures are key enablers for dynamic TDD.

One may find works on dynamic TDD that mainly focus on optimizing the UL-DL configuration ([2], [6], [7]) or the DL-to-UL switching point decision ([8]-[10]) within an LTE frame. The optimization is done either per SC or per group of SCs and, in many cases, it is constrained to the 7 LTE frame patterns predefined in LTE TDD [5]. The available techniques can be classified according to where and how such decision is taken. References like [2], [6], [7] consider a centralized and coordinated (i.e. cluster-specific) decision, where SCs are divided into isolated groups of SCs (or clusters) and the same UL-DL configuration is used within the cluster. This way, DLto-UL and UL-to-DL intra-cluster interference is avoided but the flexibility of adapting to the per-SC traffic asymmetries is reduced. On the other hand, decentralized solutions are investigated in [6], [8]-[10]. In [8], the decision on the DL-toUL switching point is performed at each SC in coordination with neighboring SCs thanks to the exchange of backhaul control plane messages (prices) that take into account the traffic asymmetry of the serving users and how such decision affects to users associated to neighboring SCs. In [9], the problem is formulated as a non-cooperative game so as to minimize the overall UL and DL delay at each SC. In [6] and [10], decentralized and uncoordinated (i.e. SC-specific) solutions are evaluated, where each SC performs its own decision based on the traffic asymmetry of the serving users. It is concluded in [10] that SC-specific decisions are sufficient if interference mitigation techniques are used at the receivers. 


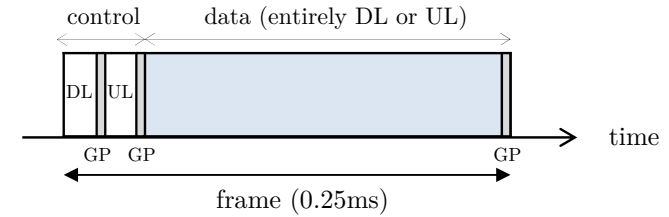

Fig. 1: Frame structure suggested for dynamic TDD in 5G [12], [13].

A similar conclusion is drawn form [6], in which interference management techniques are applied by means of coordinated scheduling and coordinated beamforming (CS-CB). They show that SC-specific decisions with CS-CB achieve a better performance than cluster-specific decisions with CS-CB, since the former can better suit traffic asymmetry conditions.

All these previous works on dynamic TDD ([2], [6]-[10]) keep the order of the transmit directions fixed (i.e. first DL and then UL) and optimize the DL-to-UL switching point or the UL-DL configuration of an LTE frame. So, they are indeed suboptimal because the transmit direction selection is constrained to the LTE frame patterns. On the contrary, authors in [11] show the positive benefits for interference reduction when the order in which UL and DL transmissions are performed at each SC is also optimized. They present a frame structure composed of two consecutive time slots. In the first slot the transmission is carried out in one direction and the reverse direction is used for the second slot (e.g. UL in first slot and DL in second slot, or the other way round). In this sense, the optimization of the transmit directions orders is investigated in conjunction with the design of linear precoding and equalization for MIMO systems. However, the main drawback of the proposed scheme in [11] is that the frame structure is equally partitioned between DL and UL and, therefore, traffic asymmetry conditions are not incorporated.

In this regard, new short-length single-direction frame structures are envisioned for $5 \mathrm{G}$ systems to meet the strict $1 \mathrm{~ms}$ latency requirement, which can not be met with the current LTE frame structure ${ }^{1}$. Low round trip times are required by some envisioned applications for $5 \mathrm{G}$ such as Tactile Internet. Following this line, a new short-length single-direction frame structure is proposed in [12] and [13] (see Fig. 1), in which the data part is entirely devoted to a single transmit direction and each SC can determine if it is used either for DL or UL. Such frame structure is very powerful for scenarios where traffic asymmetries vary drastically (as for SCNs) because of the ability to adapt the system configuration to the actual traffic and interference conditions. Also, due to the single transmit direction per frame, interference variability within the frame is avoided. Nevertheless, the key point is that, under these new frame structures envisioned for $5 \mathrm{G}$ systems, full exploitation of dynamic TDD is possible without resorting to one of the predefined UL-DL configurations in LTE TDD or focusing on the DL-to-UL switching point decision. To that end, new interference management procedures being able to determine the transmit direction in a per-frame basis are needed.

\footnotetext{
${ }^{1}$ Throughout the paper, we use the term "frame" to refer to the frame structure suggested for 5G, as shown in Fig. 1. In LTE, a $10 \mathrm{~ms}$ frame structure is defined, which is composed of 10 LTE subframes and is referenced as "LTE frame" through the paper.
}

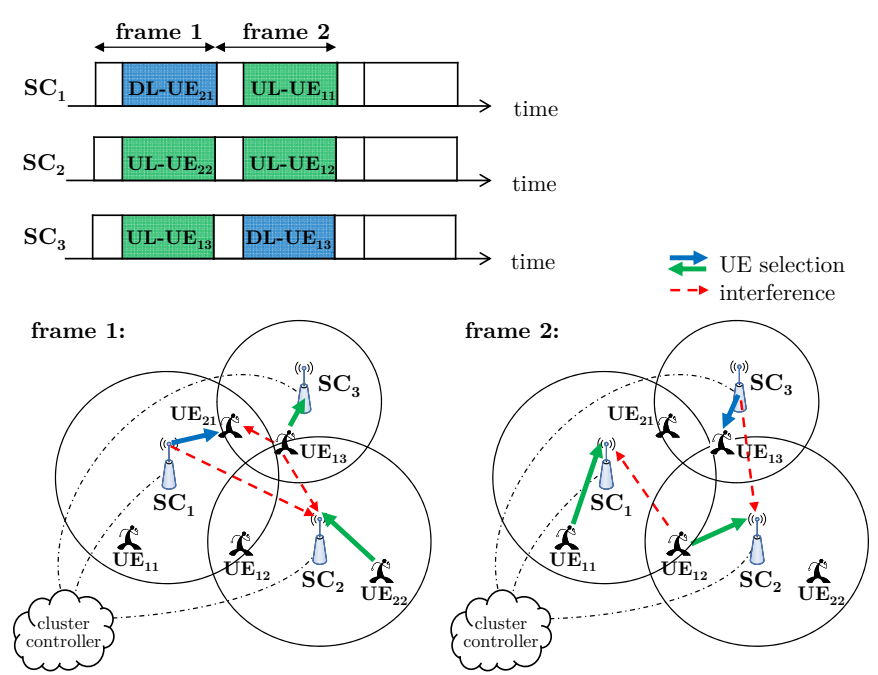

Fig. 2: Dynamic TDD in SCNs under the frame structure shown in Fig. 1. At every frame each SC schedules at most one user in one transmit direction (DL or UL). An example of the user scheduling and transmit direction selection is shown for 2 frames.

SCs are usually densified in clusters, see [14]. Thus, intracluster interference is much more limiting than inter-cluster interference, and the latter one can be usually neglected [15]. In this regard, a promising solution for $5 \mathrm{G}$ systems to perform interference management in dense SCNs is the centralizedbased concept, in which a network controller (e.g. a cluster controller) is responsible for some network functions [16] and, hence, centralized interference management techniques can be implemented for the cluster [17].

In this paper we assume a cluster-based deployment of TDD SCs and a short-length single-direction frame structure, similar to the one suggested in [12] and [13] (see Fig. 1), where each $\mathrm{SC}$ is associated to a transmit direction (DL or UL) at every frame. In this context, we propose a dynamic procedure for joint user scheduling, precoding design, and transmit direction selection that works on a per-frame basis in interfering MIMO multi-cell scenarios (see Fig. 2). A cluster controller is in charge of performing the optimization for a set of shortlength frames during which channel conditions do not vary. We face the maximization of a general utility function that takes into account the DL/UL traffic asymmetries of each user and the interference conditions in the network. First, the initial problem is decoupled through frames to achieve an on-line scheduling. Then, the per-frame problem is decomposed per $\mathrm{SC}$ by using the interference-cost concept and a direct solution is obtained (in semi-closed form for MIMO systems and in closed-form for single-input single-output (SISO) systems). Finally, an iterative algorithm is proposed to solve the perframe problem at the cluster controller.

Let us recall that in [6] the transmit direction selection is constrained to the LTE frame patterns. In addition, in [6], the optimization is done in two consecutive steps: first, the LTE frame pattern configuration is optimized (either by following a cluster-specific or a SC-specific decision) and, after that, the remaining inter-cell interference is mitigated by using CSCB. So, the transmit direction selection and the CS-CB are not jointly optimized. Thus, the dynamic procedure proposed 
in this work differs from the solutions in [6] in two main aspects: $(i)$ we do not constrain the transmit direction selection to the 7 LTE frame patterns (rather the transmit direction is optimized at every short-length frame) and (ii) we perform a joint optimization of the transmit direction and the CSCB. Consequently, although the proposed approach is also suboptimal, it provides more flexibility than those in [6].

Organization: The rest of the paper is organized as follows. In Section II the system model is presented. The problem for joint user scheduling, transmit precoding design, and transmit direction selection is formulated and decoupled through frames in Section III. Then, in Section IV the per-frame problem is decomposed into multiple subproblems (one per SC), the optimal solution for each subproblem is derived, and the iterative algorithm is presented. Finally, Section V shows the simulation results and Section VI includes concluding remarks.

Notation: In this paper, scalars are denoted by italic letters. Boldface lower-case and upper-case letters denote vectors and matrices, respectively. For given scalars $a$ and $b, \min (a, b)$ denotes the minimum between $a$ and $b,(a)^{+} \triangleq \max (0, a)$ refers to the maximum between $a$ and $0, \log _{2}(a)$ denotes the base2 logarithm, and $\ln (a)$ is the natural logarithm. For a given function $f$, the derivative of $f$ w.r.t. matrix $\mathbf{A}$ is referred to as $\frac{\delta f}{\delta \mathbf{A}}$ and $\max _{x} f$ denotes the maximum value of $f$. For a given matrix $\mathbf{A}, \mathbf{A}^{H}$ and $\mathbf{A}^{-1}$ denote the hermitian matrix and the inverse matrix, respectively. The operators $|\mathbf{A}|$ and $\operatorname{Tr}(\mathbf{A})$ refer to the determinant and the trace, respectively. Matrices $\mathbf{I}$ and $\mathbf{0}$ refer to the identity matrix and the zero matrix, respectively. $\mathbf{A}=\operatorname{diag}\left(a_{1} \ldots a_{n}\right)$ denotes an $n \times n$ diagonal matrix with values $a_{1} \ldots a_{n}$ in its diagonal and 0 in the nondiagonal elements. $\mathbf{A} \succeq \mathbf{0}$ means that matrix $\mathbf{A}$ is positive semidefinite. For given sets $\mathcal{A}$ and $\mathcal{B}$, the union is denoted by $\mathcal{A} \cup \mathcal{B}$ and $|\mathcal{A}|$ refers to the cardinality of $\mathcal{A}$. $\mathbb{C}^{m \times n}$ and $\mathbb{R}^{m \times n}$ denote the $m$ by $n$ dimensional complex space and real space, respectively. The circularly symmetric complex normal distribution is represented by $\mathcal{C N}(.,$.$) .$

\section{SYSTEM MODEL}

Consider a synchronized TDD SCN composed of clusters of SCs. We focus on interference coordination within a specific cluster and deal with intra-cluster interference, as it is done in practice (see [14, Sect. 4]), assuming that cluster formation is given by the network operator infrastructure and that intercluster interference is negligible [15]. The cluster is composed of a cluster controller that coordinates a set of $\mathcal{K} \triangleq\{1, \ldots, K\}$ SCs equipped with $M$ antennas each. Assume user-SC association is done through conventional processes (for instance, each user is associated to the $\mathrm{SC}$ that is received with largest signal-to-noise ratio), such that every $\mathrm{SC} k(k \in \mathcal{K})$ has a set of $\mathcal{I}_{k} \triangleq\left\{1_{k}, \ldots, I_{k}\right\}$ associated users with $N$ antennas each. Let $i_{k}$ denote user $i$ associated to $\mathrm{SC} k\left(i_{k} \in \mathcal{I}_{k}\right)$. The total set of users in the system (i.e. the cluster) is denoted by $\mathcal{I}=\bigcup_{k \in \mathcal{K}} \mathcal{I}_{k}$. Subindexes $\left\{i_{k}, j_{l}\right\}$ and $\{k, l\}$ are used through the paper to denote users and SCs, respectively. An example of the considered scenario is shown in Fig. 2 for $|\mathcal{K}|=3$ SCs and $|\mathcal{I}|=5$ users $\left(\left|\mathcal{I}_{1}\right|=2,\left|\mathcal{I}_{2}\right|=2,\left|\mathcal{I}_{3}\right|=1\right)$.
The short-length frame structure in [12] is assumed. Control and data planes are separated in time, as shown in Fig. 1. A short guard period (GP) is inserted between every (possible) switch of the transmit direction. The data part is entirely devoted for either UL or DL transmission, and the transmit direction in the data part (i.e. DL or UL) can vary at every frame and every SC. Through the paper, supraindex $d$ denotes the transmit direction selected at a SC in a given frame: $d=\mathrm{DL}$ refers to DL and $d=\mathrm{UL}$ refers to UL transmission.

Assume that channel state information (CSI) of all users within the cluster remains constant over a set of short-length frames $\mathcal{S} \triangleq\{1, \ldots, S\}$. Let $\mathbf{H}_{i_{k}, l} \in \mathbb{C}^{M \times N}, \quad \mathbf{H}_{l, i_{k}} \in \mathbb{C}^{N \times M}$, $\mathbf{H}_{i_{k}, j_{l}} \in \mathbb{C}^{N \times N}$, and $\mathbf{H}_{k, l} \in \mathbb{C}^{M \times M}$ denote the MIMO channel matrix (including pathloss, shadowing, and small-scale fading) from user $i_{k}$ to SC $l$, from SC $l$ to user $i_{k}$, from user $i_{k}$ to user $j_{l}$, and from SC $k$ to SC $l$, respectively.

It is considered that at every frame $(s=1, \ldots, S)$, at most one user is scheduled in one transmit direction (either DL or UL) at each SC (see Fig. 2).

Therefore, the cluster controller has two main functions:

- gathers CSI of all users in the cluster both in DL and UL transmit directions (which are valid for the whole set of short-length frames $\mathcal{S}$ ) and

- is in charge of performing the dynamic resource management for SCs in the cluster, which involves the joint optimization of the user scheduling, transmit precoding design, and transmit direction selection per frame.

So, the resource management is applied on a per-frame basis while acquisition of CSI is done every $S$ frames.

Assuming narrow-band transmissions, the equivalent baseband signal observed at user $i_{k}$ in frame $s$ (assuming DL is selected at SC $k$ in frame $s$ and no other user is served by SC $k$ at that frame) is expressed as:

$$
\mathbf{y}_{i_{k}, s}^{\mathrm{DL}}=\mathbf{H}_{k, i_{k}} \mathbf{x}_{i_{k}, s}^{\mathrm{DL}}+\underbrace{\sum_{\substack{j_{l} \in \mathcal{I}_{l} \\ l \neq k}} \mathbf{H}_{l, i_{k}} \mathbf{x}_{j_{l}, s}^{\mathrm{DL}}}_{\text {DL-to-DL interference }}+\underbrace{\sum_{\substack{j_{l} \in \mathcal{I}_{l} \\ l \neq k}} \mathbf{H}_{j_{l}, i_{k}} \mathbf{x}_{j_{l}, s}^{\mathrm{UL}}}_{\text {UL-to-DL interference }}+\mathbf{z}_{i_{k}, s}^{\mathrm{DL}},
$$

where $\mathbf{x}_{i_{k}, s}^{\mathrm{DL}} \in \mathbb{C}^{M \times 1}, \quad \mathbf{x}_{j_{l}, s}^{\mathrm{DL}} \in \mathbb{C}^{M \times 1}, \quad \mathbf{x}_{j_{l}, s}^{\mathrm{UL}} \in \mathbb{C}^{N \times 1}$ denote the transmitted signal at SC $k$ towards user $i_{k}$, at SC $l$ towards user $j_{l}$, at user $j_{l}$ towards SC $l$, respectively, and $\mathbf{z}_{i_{k}, s}^{\mathrm{DL}}$ refers to the received noise vector at user $i_{k}$ in frame $s$. Similarly, the equivalent baseband signal observed at SC $k$ in frame $s$ (assuming UL is selected at SC $k$ in frame $s$ and only user $i_{k}$ transmits to it at that frame) is:

$$
\mathbf{y}_{i_{k}, s}^{\mathrm{UL}}=\mathbf{H}_{i_{k}, k} \mathbf{x}_{i_{k}, s}^{\mathrm{UL}}+\underbrace{\sum_{\substack{j_{l} \in \mathcal{I}_{l} \\ l \neq k}} \mathbf{H}_{l, k} \mathbf{x}_{j_{l}, s}^{\mathrm{DL}}}_{\text {DL-to-UL interference }}+\underbrace{\sum_{\substack{j_{l} \in \mathcal{I}_{l} \\ l \neq k}} \mathbf{H}_{j_{l}, k} \mathbf{x}_{j_{l}, s}^{\mathrm{UL}}}_{\text {UL-to-UL interference }}+\mathbf{z}_{k, s}^{\mathrm{UL}},
$$

where $\mathbf{x}_{i_{k}, s}^{\mathrm{UL}} \in \mathbb{C}^{N \times 1}$ denotes the transmitted signal at user $i_{k}$ towards SC $k$ and $\mathbf{z}_{k, s}^{\mathrm{UL}}$ refers to the received noise vector at $\mathrm{SC} k$ in frame $s$. It is assumed that $\mathbf{z}_{k, s}^{\mathrm{UL}} \sim \mathcal{C N}\left(0, \sigma_{k}^{2}\right)$, $\mathbf{z}_{i_{k}, s}^{\mathrm{DL}} \sim \mathcal{C N}\left(0, \sigma_{i_{k}}^{2}\right), \forall s$, and that all $\left\{\mathbf{z}_{k, s}^{\mathrm{UL}}\right\}$ and $\left\{\mathbf{z}_{i_{k}, s}^{\mathrm{DL}}\right\}$ are independent. Note that, under dynamic TDD, new kinds of inter-cell interference arise as compared to conventional oneway transmissions. In a DL transmission, DL-to-DL and UL- 
to-DL interference might be received (see (1)). In contrast, for an UL transmission, DL-to-UL and UL-to-UL interference may appear (as shown in (2)).

We assume independent encoding across the different SCs and users and that a Gaussian codebook is used at each SC and each user, i.e.:

$$
\begin{aligned}
& \mathbf{x}_{i_{k}, s}^{\mathrm{DL}} \sim \mathcal{C N}\left(\mathbf{0}, \mathbf{Q}_{i_{k}, s}^{\mathrm{DL}}\right), \\
& \mathbf{x}_{i_{k}, s}^{\mathrm{UL}} \sim \mathcal{C N}\left(\mathbf{0}, \mathbf{Q}_{i_{k}, s}^{\mathrm{UL}}\right),
\end{aligned}
$$

where $\mathbf{Q}_{i_{k}, s}^{\mathrm{DL}}$ denotes the transmit covariance matrix for SC $k$ to serve user $i_{k}$ in frame $s\left(\mathbf{Q}_{i_{k}, s}^{\mathrm{DL}} \in \mathbb{C}^{M \times M}\right.$ and $\left.\mathbf{Q}_{i_{k}, s}^{\mathrm{DL}} \succeq \mathbf{0}\right)$ and $\mathbf{Q}_{i_{k}, s}^{\mathrm{UL}}$ refers to the transmit covariance matrix for user $i_{k}$ to transmit towards SC $k$ in frame $s\left(\mathbf{Q}_{i_{k}, s}^{\mathrm{UL}} \in \mathbb{C}^{N \times N}\right.$ and $\left.\mathbf{Q}_{i_{k}, s}^{\mathrm{UL}} \succeq \mathbf{0}\right)$.

The optimization variables are the transmit covariance matrices, $\left\{\mathbf{Q}_{i_{k}, s}^{\mathrm{DL}}, \mathbf{Q}_{i_{k}, s}^{\mathrm{UL}}\right\}_{\forall i_{k}, k, s}$, which contain information of precoding design, power control, user scheduling, and transmit direction selection as stated in the following.

- The precoder design is directly obtained through the eigenvalue decomposition of the transmit covariances matrices, i.e. $\mathbf{Q}_{i_{k}, s}^{d}=\mathbf{U}_{i_{k}, s}^{d} \mathbf{P}_{i_{k}, s}^{d}\left(\mathbf{U}_{i_{k}, s}^{d}\right)^{-1}$, where $\mathbf{U}_{i_{k}, s}^{d}$ is the unitary transmit precoding matrix.

- The power employed by SC $k$ to serve user $i_{k}$ in frame $s$ is given by: $\operatorname{Tr}\left(\mathbf{Q}_{i_{k}, s}^{\mathrm{DL}}\right)=\operatorname{Tr}\left(\mathbf{P}_{i_{k}, s}^{\mathrm{DL}}\right)$. Similarly, the power used by user $i_{k}$ to transmit towards SC $k$ in frame $s$ is obtained as: $\operatorname{Tr}\left(\mathbf{Q}_{i_{k}, s}^{\mathrm{UL}}\right)=\operatorname{Tr}\left(\mathbf{P}_{i_{k}, s}^{\mathrm{UL}}\right)$.

- The user scheduling and transmit direction selection are contained in $\left\{\mathbf{Q}_{i_{k}, s}^{\mathrm{DL}}, \mathbf{Q}_{i_{k}, s}^{\mathrm{UL}}\right\}_{\forall i_{k}, k, s}$ as follows:

- if $\operatorname{Tr}\left(\mathbf{Q}_{i_{k}, s}^{d}\right)>0$, then user $i_{k}$ is scheduled and transmit direction $d$ is selected at SC $k$ in frame $s$,

- if $\operatorname{Tr}\left(\mathbf{Q}_{i_{k}, s}^{d}\right)=0$ then user $i_{k}$ is not scheduled in transmit direction $d$ at SC $k$ in frame $s$.

This intrinsic information is valid provided that we impose certain constraints over the transmit covariance matrices $\left\{\mathbf{Q}_{i_{k}, s}^{\mathrm{DL}}, \mathbf{Q}_{i_{k}, s}^{\mathrm{UL}}\right\}_{\forall i_{k}, k, s}$.

The constraint that at most one user in one transmit direction is selected at every SC in each frame can be imposed directly over optimization variables $\left\{\mathbf{Q}_{i_{k}, s}^{\mathrm{DL}}, \mathbf{Q}_{i_{k}, s}^{\mathrm{UL}}\right\}_{\forall i_{k}, k, s}$ as:

$$
\sum_{i_{k} \in \mathcal{I}_{k}}\left(\mathbb{1}\left\{\mathbf{Q}_{i_{k}, s}^{\mathrm{DL}}\right\}+\mathbb{1}\left\{\mathbf{Q}_{i_{k}, s}^{\mathrm{UL}}\right\}\right) \leq 1 \quad \forall k, s,
$$

where $\mathbb{1}\left\{\mathbf{Q}_{i_{k}, s}^{d}\right\}$ denotes the indicator function over transmit covariance matrix $\mathbf{Q}_{i_{k}, s}^{d}$ :

$$
\mathbb{1}\left\{\mathbf{Q}_{i_{k}, s}^{d}\right\}=\left\{\begin{array}{ll}
1 & \text { if } \mathbf{Q}_{i_{k}, s}^{d} \neq \mathbf{0} \\
0 & \text { if } \mathbf{Q}_{i_{k}, s}^{d}=\mathbf{0}
\end{array} .\right.
$$

Constraint in (5) is required to be consistent with the formulation presented in (1)-(2). Otherwise, in case multiple users could be simultaneously served at a frame and within the same frequency resource, intra-cell interference should be considered in the signal model.

Usually, in the literature, designers deal with integer variables that indicate the user scheduling and transmit direction selection (see [11]), in addition to optimizing the transmit precoding matrices. Differently, thanks to the presented formulation, a single set of variables (i.e. $\left\{\mathbf{Q}_{i_{k}, s}^{\mathrm{DL}}, \mathbf{Q}_{i_{k}, s}^{\mathrm{UL}}\right\}_{\forall i_{k}, k, s}$ ) has to be optimized. In this sense, constraints in (5) replace the integer constraints that are usually included through a set of integer variables that take 0 or 1 value [11]. The key point does not rely on the reduction of the sets of variables to be optimized, but rather on the fact that working with a single set of non-integer variables (i.e. $\left\{\mathbf{Q}_{i_{k}, s}^{d}\right\}$ ) will allow us to decompose the global problem until a point where the decomposed problems can be optimally solved (as it will be shown in Sections III and IV).

Under this setting, assuming that interference is treated as Gaussian noise at receivers, the achievable rates for DL and UL transmissions, respectively, of user $i_{k}$ in frame $s, r_{i_{k}, s}^{\mathrm{DL}}$, $r_{i_{k}, s}^{\mathrm{UL}}$, are given by:

$$
\begin{aligned}
& r_{i_{k}, s}^{\mathrm{DL}}=\log _{2}\left|\mathbf{I}+\mathbf{H}_{k, i_{k}} \mathbf{Q}_{i_{k}, s}^{\mathrm{DL}} \mathbf{H}_{k, i_{k}}^{H}\left(\mathbf{N}_{i_{k}, s}^{\mathrm{DL}}\right)^{-1}\right|, \\
& r_{i_{k}, s}^{\mathrm{UL}}=\log _{2}\left|\mathbf{I}+\mathbf{H}_{i_{k}, k} \mathbf{Q}_{i_{k}, s}^{\mathrm{UL}} \mathbf{H}_{i_{k}, k}^{H}\left(\mathbf{N}_{i_{k}, s}^{\mathrm{UL}}\right)^{-1}\right|,
\end{aligned}
$$

where $\mathbf{N}_{i_{k}, s}^{\mathrm{DL}}$ is the covariance matrix of the noise-plusinterference received at user $i_{k}$ when SC $k$ transmits in frame $s$ and $\mathbf{N}_{i_{k}, s}^{\mathrm{UL}}$ denotes the covariance matrix of the noise-plusinterference received at SC $k$ when user $i_{k}$ transmits in frame $s$ :

$$
\begin{aligned}
& \mathbf{N}_{i_{k}, s}^{\mathrm{DL}}=\sigma_{i_{k}}^{2} \mathbf{I}+\sum_{l \neq k, j_{l} \in \mathcal{I}_{l}} \mathbf{H}_{l, i_{k}} \mathbf{Q}_{j_{l}, s}^{\mathrm{DL}} \mathbf{H}_{l, i_{k}}^{H}+\mathbf{H}_{j_{l}, i_{k}} \mathbf{Q}_{j_{l}, s}^{\mathrm{UL}} \mathbf{H}_{j_{l}, i_{k}}^{H}, \\
& \mathbf{N}_{i_{k}, s}^{\mathrm{UL}}=\sigma_{k}^{2} \mathbf{I}+\sum_{l \neq k, j_{l} \in \mathcal{I}_{l}} \mathbf{H}_{l, k} \mathbf{Q}_{j_{l}, s}^{\mathrm{DL}} \mathbf{H}_{l, k}^{H}+\mathbf{H}_{j_{l}, k} \mathbf{Q}_{j_{l}, s}^{\mathrm{UL}} \mathbf{H}_{j_{l}, k}^{H} .
\end{aligned}
$$

As shown in (9)-(10), interference received in DL and UL depends on the user scheduling, precoding design, and transmit direction selected at neighbor SCs in frame $s$.

\section{Problem Formulation}

The problem for joint user scheduling, precoding design, and transmit direction selection is formulated by following the maximization of a general utility function that takes into account the traffic asymmetries and interference conditions in the network under the constraints that at most one user in one transmit direction is selected at each SC in every frame (see (5)) and that a maximum power is available for DL and UL transmission at SCs and users, respectively:

$$
\begin{aligned}
\left(\mathrm{P}_{\text {global }}\right): \operatorname{cox}_{\substack{\mathbf{Q}_{i_{k}}^{d}, s \\
\forall i_{k}, k, s, d}} \sum_{\substack{k \in \mathcal{K} \\
i_{k} \in \mathcal{I}_{k}}}\left(a_{i_{k}} u\left(\bar{R}_{i_{k}}^{\mathrm{DL}}\right)+\left(1-a_{i_{k}}\right) u\left(\bar{R}_{i_{k}}^{\mathrm{UL}}\right)\right) \\
\text { subject to }\left\{\begin{array}{l}
\sum_{i_{k} \in \mathcal{I}_{k}}\left(\mathbb{1}\left\{\mathbf{Q}_{i_{k}, s}^{\mathrm{DL}}\right\}+\mathbb{1}\left\{\mathbf{Q}_{i_{k}, s}^{\mathrm{UL}}\right\}\right) \leq 1 \forall k, s \\
\operatorname{Tr}\left(\mathbf{Q}_{i_{k}, s}^{\mathrm{DL}}\right) \leq P_{\mathrm{SC}}^{\max } \quad \forall i_{k}, k, s \\
\operatorname{Tr}\left(\mathbf{Q}_{i_{k}, s}^{\mathrm{UL}}\right) \leq P_{\mathrm{UE}}^{\max } \quad \forall i_{k}, k, s
\end{array}\right.
\end{aligned}
$$

where $0 \leq a_{i_{k}} \leq 1$ is related to the DL-UL data traffic asymmetry of user $i_{k}, u(z)$ is a concave and monotonically increasing utility function on the interval $z \in[0, \infty), P_{\mathrm{SC}}^{\max }$ is the available power at SCs, $P_{\mathrm{UE}}^{\max }$ is the available power at users, and $\bar{R}_{i_{k}}^{d}$ is the average rate of user $i_{k}$ in transmit direction $d$ $(d=\{\mathrm{DL}, \mathrm{UL}\})$ over the set of frames $\mathcal{S}$ :

$$
\bar{R}_{i_{k}}^{d}=\frac{1}{|\mathcal{S}|} \sum_{s \in \mathcal{S}} r_{i_{k}, s}^{d} .
$$


A classical choice for the utility function in (11) is $u(z)=\log (z)$, which guarantees proportional fairness in the system [18]. Even though, alternative choices that are suitable for different types of applications can be found in [19] (e.g. $u(z)=z$ for sum rate maximization or $u(z)=-1 / z$ for harmonic mean fairness).

Practical scheduling algorithms welcome on-line solutions that are capable of adapting weights to obtain desirable DL/UL user fairness [20]. In this sense, and without loss of generality, the average rate of user $i_{k}$ in transmit direction $d$ can be updated at every frame using standard stochastic approximation recursions (see [21]):

$$
\bar{R}_{i_{k}, s+1}^{d}=\bar{R}_{i_{k}, s}^{d}+\alpha_{s}\left(r_{i_{k}, s}^{d}-\bar{R}_{i_{k}, s}^{d}\right),
$$

where $\alpha_{s}$ is a step-size that can be either asymptotically vanishing (e.g. $\left.\alpha_{s}=1 / s\right)$ or constant $\left(\alpha_{s}=1 /|\mathcal{S}|\right), \bar{R}_{i_{k}, s}^{d}$ is the average rate of user $i_{k}$ in transmit direction $d$ in previous frames, and $r_{i_{k}, s}^{d}$ is the rate given to user $i_{k}$ in transmit direction $d$ in frame $s$ (see (7)-(8)). Using Taylor's expansion with step-size $\alpha_{s}$ sufficiently small over the on-line averaging performed in the recursion in (13), we get [20]:

$$
u\left(\bar{R}_{i_{k}, s+1}^{d}\right) \approx u\left(\bar{R}_{i_{k}, s}^{d}\right)+\frac{\delta u\left(\bar{R}_{i_{k}, s}^{d}\right)}{\delta \bar{R}_{i_{k}, s}^{d}} \alpha_{s}\left(r_{i_{k}, s}^{d}-\bar{R}_{i_{k}, s}^{d}\right) .
$$

Therefore, since $\bar{R}_{i_{k}, s}^{d}, u\left(\bar{R}_{i_{k}, s}^{d}\right)$ and $\frac{\delta u\left(\bar{R}_{i_{k}, s}^{d}\right)}{\delta \bar{R}_{i_{k}, s}^{d}}$ are available in frame $s$, maximizing $\left(\mathrm{P}_{\text {global }}\right)$ in (11) with $u\left(\bar{R}_{i_{k}, s+1}^{d}\right)$ in (14) instead of $u\left(\bar{R}_{i_{k}}^{d}\right)$ in (11) reduces to the following maximization problem that has to be solved for every frame and corresponds to a weighted sum of the achievable rates. The on-line solution for frame $s$ is obtained from:

$$
\begin{aligned}
\left(\mathrm{P}_{\text {frame }}^{s}\right): \underset{\substack{\left.\operatorname{maximize}_{i_{k}, s}^{d} \succeq \mathbf{0}\right\} \\
\forall i_{k}, k, d}}{\operatorname{man}} \sum_{\substack{i_{k} \in \mathcal{\mathcal { I }} \\
\text { subject to }}}\left(\mu_{i_{k}, s}^{\mathrm{DL}} r_{i_{k}, s}^{\mathrm{DL}}+\mu_{i_{k}, s}^{\mathrm{UL}} s_{i_{k}, s}^{\mathrm{UL}}\right) \\
\left\{\begin{array}{l}
\sum_{i_{k} \in \mathcal{I}_{k}}\left(\mathbb{1}\left\{\mathbf{Q}_{i_{k}, s}^{\mathrm{DL}}\right\}+\mathbb{1}\left\{\mathbf{Q}_{i_{k}, s}^{\mathrm{UL}}\right\}\right) \leq 1 \forall k \\
\operatorname{Tr}\left(\mathbf{Q}_{i_{k}, s}^{\mathrm{DL}}\right) \leq P_{\mathrm{SC}}^{\max } \quad \forall i_{k}, k \\
\operatorname{Tr}\left(\mathbf{Q}_{i_{k}, s}^{\mathrm{UL}}\right) \leq P_{\mathrm{UE}}^{\max } \quad \forall i_{k}, k
\end{array}\right.
\end{aligned}
$$

where $\mu_{i_{k}, s}^{d}$ is a fixed weight associated to user $i_{k}$ in transmit direction $d$ in frame $s . \mu_{i_{k}, s}^{d}$ depends on the traffic asymmetry $a_{i_{k}}$ (see (11)) and on the derivative of the utility function $u(z)$ adopted for problem $\left(\mathrm{P}_{\text {global }}\right)$ in (11) (as derived from (14)):

$$
\mu_{i_{k}, s}^{\mathrm{DL}}=a_{i_{k}} \frac{\delta u\left(\bar{R}_{i_{k}, s}^{\mathrm{DL}}\right)}{\delta \bar{R}_{i_{k}, s}^{\mathrm{DL}}}, \quad \mu_{i_{k}, s}^{\mathrm{UL}}=\left(1-a_{i_{k}}\right) \frac{\delta u\left(\bar{R}_{i_{k}, s}^{\mathrm{UL}}\right)}{\delta \bar{R}_{i_{k}, s}^{\mathrm{UL}}} .
$$

For example, in case $u(z)=\log (z)$ then $\frac{\delta u\left(\bar{R}_{i_{k}, s}^{d}\right)}{\delta \bar{R}_{i_{k}, s}^{d}}=1 / \bar{R}_{i_{k}, s}^{d}$, such that a modified version of the well-known proportional fair criterion [18] would be obtained ${ }^{2}$. However, the formulation of problem $\left(\mathrm{P}_{\text {global }}\right)$ in (11) allows accommodating general utility functions that correspond to different traffic types and quality-of-service requirements (as best effort, non-real-time, and real-time services) whereas only the design of the weights

\footnotetext{
${ }^{2}$ The modification comes from the inclusion of the traffic asymmetry conditions, i.e. $a_{i_{k}}$, see (16).
}

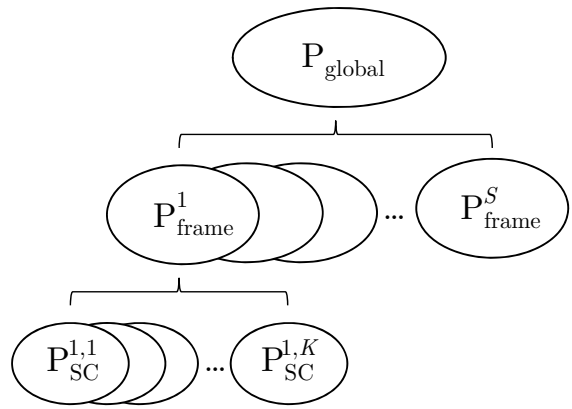

Fig. 3: Decoupling of the global problem ( $\left.\mathrm{P}_{\text {global }}\right)$ in (11) into $S$ problems (one per frame): $\left(\mathrm{P}_{\text {frame }}^{s}\right)$ in (15). Then, problem $\left(\mathrm{P}_{\text {frame }}^{s}\right)$ is decomposed into $K$ subproblems (one per SC): $\left(\mathrm{P}_{\mathrm{SC}}^{s, k}\right)$ in $(21)$, which are solved in parallel at the cluster controller.

per user and transmit direction $\mu_{i_{k}, s}^{d}$ for problem $\left(\mathrm{P}_{\text {frame }}^{s}\right)$ in (15) are affected (see details in [20]).

The objective function of problem $\left(\mathrm{P}_{\text {frame }}^{s}\right)$ in (15) is nonconvex due to interference (see (7)-(8)) and some constraints are non-continuous. Therefore, finding the global optimum is a challenging task. In this regard, Section IV proposes an efficient algorithm that yields a local optimum solution to problem $\left(\mathrm{P}_{\text {frame }}^{s}\right)$ in (15).

After optimizing problem $\left(\mathrm{P}_{\text {frame }}^{s}\right)$ in (15) for a given frame, the weights per user and transmit direction, $\mu_{i_{k}, s+1}^{\mathrm{DL}}, \mu_{i_{k}, s+1}^{\mathrm{UL}}$, have to be updated and problem $\left(\mathrm{P}_{\text {frame }}^{s+1}\right)$ in (15) can be subsequently solved for frame $s+1$. This provides an on-line solution, as the user scheduling, precoding design, and transmit direction selection in frame $s$ might impact on the weights used in the subsequent frame $s+1$. Note that the whole set of per-frame problems $\left(\mathrm{P}_{\text {frame }}^{s}\right)$ in (15), $\forall s \in \mathcal{S}$, can be solved at every frame or all together at the beginning of the set of frames if channel coherence time allows it.

Finally, let us remark that the sequence of average rates $\bar{R}_{i_{k}, s}^{d}$ obtained with the on-line solution converges as $s \rightarrow \infty$ to the sequence of average rates $\bar{R}_{i_{k}}^{d}$ that solves problem ( $\mathrm{P}_{\text {global }}$ ) in (11), see [22], [23]. When the number of frames is finite, convergence of both sequences to the same solution cannot be guaranteed. Anyway, on-line solutions are desirable for practical scheduling algorithms. So, from now on, we focus on solving the per-frame problem $\left(\mathrm{P}_{\text {frame }}^{s}\right)$ in (15).

\section{Joint User Scheduling, Precoding Design And Transmit Direction Selection to Solve (15)}

In this section we propose an algorithm to solve the perframe problem $\left(\mathrm{P}_{\text {frame }}^{s}\right)$ in (15) and hence obtaining the user scheduling, precoding design, and transmit direction selection for all SCs in frame $s$. Although the per-frame problem $\left(\mathrm{P}_{\text {frame }}^{s}\right)$ in (15) is non-convex, we exploit decomposition techniques and the interference-cost concept presented in [24] to solve it. To do so, the per-frame problem $\left(\mathrm{P}_{\text {frame }}^{s}\right)$ in (15) is decomposed into $K$ subproblems (one subproblem per SC) and the solution is obtained by solving them iteratively at the cluster controller. The subproblems are obtained by replacing the interference that renders the objective function of problem $\left(\mathrm{P}_{\text {frame }}^{s}\right)$ in (15) non-convex by linear approximations, as it is done in [24][26]. Fig. 3 shows the whole decomposition. 


\section{A. Decomposition of (15)}

Let us define the interference-cost (or price) matrices [24], [25] related to the impact of selecting user $i_{k}$ at SC $k$ in transmit direction $d=\mathrm{DL}$ and $d=\mathrm{UL}$, respectively, over the neighboring SCs/users $\left(\forall l \neq k, \forall j_{l} \in \mathcal{I}_{l}\right)$ :

$$
\begin{aligned}
& \boldsymbol{\Upsilon}_{i_{k}, s}^{\mathrm{DL}}=-\sum_{l \neq k, j_{l} \in \mathcal{I}_{l}}\left(\mu_{j_{l}, s}^{\mathrm{DL}} \frac{\delta r_{j_{l}, s}^{\mathrm{DL}}}{\delta \mathbf{Q}_{i_{k}, s}^{\mathrm{DL}}}+\mu_{j_{l}, s}^{\mathrm{UL}} \frac{\delta r_{j_{l}, s}^{\mathrm{UL}}}{\delta \mathbf{Q}_{i_{k}, s}^{\mathrm{DL}}}\right), \\
& \boldsymbol{\Upsilon}_{i_{k}, s}^{\mathrm{UL}}=-\sum_{l \neq k, j_{l} \in \mathcal{I}_{l}}\left(\mu_{j_{l}, s}^{\mathrm{DL}} \frac{\delta r_{j_{l}, s}^{\mathrm{DL}}}{\delta \mathbf{Q}_{i_{k}, s}^{\mathrm{UL}}}+\mu_{j_{l}, s}^{\mathrm{UL}} \frac{\delta r_{j_{l}, s}^{\mathrm{UL}}}{\delta \mathbf{Q}_{i_{k}, s}^{\mathrm{UL}}}\right),
\end{aligned}
$$

where $\frac{\delta r_{j_{l}, s}^{\mathrm{DL}}}{\delta \mathbf{Q}_{i_{k}, s}^{\mathrm{DL}}}$ denotes a matrix corresponding to the derivative of scalar function $r_{j_{l}, s}^{\mathrm{DL}}$ w.r.t. transmit covariance matrix $\mathbf{Q}_{i_{k}, s}^{\mathrm{DL}}$. The derivatives can be obtained using the framework developed in [27] for complex-valued matrix differentiation. For example, $\frac{\delta r_{j_{l}, s}^{\mathrm{DL}}}{\delta \mathbf{Q}_{i_{k}, s}^{\mathrm{DL}}}$ and $\frac{\delta r_{j_{l}, s}^{\mathrm{DL}}}{\delta \mathbf{Q}_{i_{k}, s}^{\mathrm{UL}}}$ are given by:

$$
\begin{aligned}
\frac{\delta r_{j_{l}, s}^{\mathrm{DL}}}{\delta \mathbf{Q}_{i_{k}, s}^{\mathrm{DL}}} & =\frac{1}{\ln (2)} \mathbf{H}_{k, j_{l}}^{H} \mathbf{Z}_{j_{l}, s}^{\mathrm{DL}} \mathbf{H}_{k, j_{l}}, \\
\frac{\delta r_{j_{l}, s}^{\mathrm{DL}}}{\delta \mathbf{Q}_{i_{k}, s}^{\mathrm{UL}}} & =\frac{1}{\ln (2)} \mathbf{H}_{i_{k}, j_{l}}^{H} \mathbf{Z}_{j_{l}, s}^{\mathrm{DL}} \mathbf{H}_{i_{k}, j_{l}},
\end{aligned}
$$

where

$$
\mathbf{Z}_{j_{l}, s}^{\mathrm{DL}}=\left(\mathbf{N}_{j_{l}, s}^{\mathrm{DL}}+\mathbf{H}_{l, j_{l}} \mathbf{Q}_{j_{l}, s}^{\mathrm{DL}} \mathbf{H}_{l, j_{l}}^{H}\right)^{-1}-\left(\mathbf{N}_{j_{l}, s}^{\mathrm{DL}}\right)^{-1} .
$$

The remaining derivatives $\frac{\delta r_{j_{l}, s}^{\mathrm{UL}}}{\delta \mathbf{Q}_{i_{k}, s}^{\mathrm{DL}}}$ and $\frac{\delta r_{j_{l}, s}^{\mathrm{UL}}}{\delta \mathbf{Q}_{i_{k}, s}^{\mathrm{UL}}}$ can be similarly obtained, but are omitted for brevity. Interference-cost matrices $\Upsilon_{i_{k}, s}^{\mathrm{DL}}$ and $\boldsymbol{\Upsilon}_{i_{k}, s}^{\mathrm{UL}}$ in (17)-(18) allow replacing by linear approximations the interference generated by the transmission towards/from users at SC $k$ over neighboring SCs/users [24].

Therefore, as the constraints of problem $\left(\mathrm{P}_{\text {frame }}^{S}\right)$ in (15) can be directly decomposed per SC and the objective function of problem $\left(\mathrm{P}_{\text {frame }}^{s}\right)$ in (15) can be decoupled thanks to the interference-cost concept, it is possible to approximate the perframe problem $\left(\mathrm{P}_{\text {frame }}^{s}\right)$ in (15) by a set of $K$ subproblems (one per SC). Further details are included in Appendix A.

For fixed interference-cost matrices $\left(\Upsilon_{i_{k}, s}^{\mathrm{DL}}, \Upsilon_{i_{k}, s}^{\mathrm{UL}}, \forall i_{k} \in \mathcal{I}_{k}\right.$, in (17)-(18)) and fixed interference-plus-noise covariance matrices $\left(\mathbf{N}_{i_{k}, s}^{\mathrm{DL}}, \mathbf{N}_{i_{k}, s}^{\mathrm{UL}}, \forall i_{k} \in \mathcal{I}_{k}\right.$, in (9)-(10)), the subproblem corresponding to $\mathrm{SC} k$ is:

$$
\begin{aligned}
& \left(\mathrm{P}_{\mathrm{SC}}^{s, k}\right): \underset{\substack{\left\{\mathbf{Q}_{i_{k}, s}^{d} \succeq \mathbf{0}\right\} \\
\forall i_{k} \in \mathcal{I}_{k}, d}}{\operatorname{maximize}} \sum_{i_{k} \in \mathcal{I}_{k}}\left(\mu_{i_{k}, s}^{\mathrm{DL}} r_{i_{k}, s}^{\mathrm{DL}}\left(\mathbf{Q}_{i_{k}, s}^{\mathrm{DL}}\right)-\operatorname{Tr}\left(\mathbf{Q}_{i_{k}, s}^{\mathrm{DL}} \mathbf{\Upsilon}_{i_{k}, s}^{\mathrm{DL}}\right)\right) \\
& +\sum_{i_{k} \in \mathcal{I}_{k}}\left(\mu_{i_{k}, s}^{\mathrm{UL}} r_{i_{k}, s}^{\mathrm{UL}}\left(\mathbf{Q}_{i_{k}, s}^{\mathrm{UL}}\right)-\operatorname{Tr}\left(\mathbf{Q}_{i_{k}, s}^{\mathrm{UL}} \mathbf{\Upsilon}_{i_{k}, s}^{\mathrm{UL}}\right)\right) \\
& \text { subject to }\left\{\begin{array}{lc}
\sum_{i_{k} \in \mathcal{I}_{k}}\left(\mathbb{1}\left\{\mathbf{Q}_{i_{k}, s}^{\mathrm{DL}}\right\}+\mathbb{1}\left\{\mathbf{Q}_{i_{k}, s}^{\mathrm{UL}}\right\}\right) \leq 1 \\
\operatorname{Tr}\left(\mathbf{Q}_{i_{k}, s}^{\mathrm{DL}}\right) \leq P_{\mathrm{SC}}^{\max } & \forall i_{k} \in \mathcal{I}_{k} \\
\operatorname{Tr}\left(\mathbf{Q}_{i_{k}, s}^{\mathrm{UL}}\right) \leq P_{\mathrm{UE}}^{\max } & \forall i_{k} \in \mathcal{I}_{k}
\end{array}\right.
\end{aligned}
$$

where $r_{i_{k}, s}^{\mathrm{DL}}\left(\mathbf{Q}_{i_{k}, s}^{\mathrm{DL}}\right)$ and $r_{i_{k}, s}^{\mathrm{UL}}\left(\mathbf{Q}_{i_{k}, s}^{\mathrm{UL}}\right)$ are the achievable rates in (7)-(8) for fixed $\mathbf{N}_{i_{k}, s}^{\mathrm{DL}}$ and $\mathbf{N}_{i_{k}, s}^{\mathrm{UL}} . \mathbf{\Upsilon}_{i_{k}, s}^{\mathrm{DL}}, \mathbf{\Upsilon}_{i_{k}, s}^{\mathrm{UL}}$ in (17)(18) and $\mathbf{N}_{i_{k}, s}^{\mathrm{DL}}, \mathbf{N}_{i_{k}, s}^{\mathrm{UL}}$ in (9)-(10), $\forall i_{k} \in \mathcal{I}_{k}$, are computed at each iteration with the values of $\left\{\mathbf{Q}_{i_{k}, s}^{d}\right\}$ obtained from the previous iteration (see Appendix A). The trace terms in the objective function of (21) play the role of an interference tax, discouraging selfish behavior of user $i_{k}$ in transmit direction $d$ in case $\boldsymbol{\Upsilon}_{i_{k}, s}^{d} \neq \mathbf{0}$. Otherwise, in case $\boldsymbol{\Upsilon}_{i_{k}, s}^{d}=\mathbf{0}$, user $i_{k}$ in transmit direction $d$ would just want to maximize its own achievable rate $r_{i_{k}, s}^{d}\left(\mathbf{Q}_{i_{k}, s}^{d}\right)$.

Note that $r_{i_{k}, s}^{d}\left(\mathbf{Q}_{i_{k}, s}^{d}\right)$ in (21) is concave w.r.t. $\mathbf{Q}_{i_{k}, s}^{d}$ and $\operatorname{Tr}\left(\mathbf{Q}_{i_{k}, s}^{d} \boldsymbol{\Upsilon}_{i_{k}, s}^{d}\right)$ is linear. Then, as the objective function of subproblem $\left(\mathrm{P}_{\mathrm{SC}}^{s, k}\right)$ in $(21)$ is separable among users and transmit directions, the objective function of subproblem $\left(\mathrm{P}_{\mathrm{SC}}^{s, k}\right)$ in (21) is concave w.r.t. $\left\{\mathbf{Q}_{i_{k}, s}^{d}\right\}_{\forall i_{k} \in \mathcal{I}_{k}, d}$. Thus, after the whole steps of decomposition (see Fig. 3), we only have to deal with the non-continuous constraint (i.e. first constraint in (21)).

\section{B. Solution to (21)}

In case the first constraint in (21) was removed, subproblem $\left(\mathrm{P}_{\mathrm{SC}}^{s, k}\right)$ in $(21)$ would be a convex optimization problem and an optimal solution for $\left\{\mathbf{Q}_{i_{k}, s}^{d}\right\}_{\forall i_{k} \in \mathcal{I}_{k}, d}$ would exist. Even though, the first constraint of subproblem $\left(\mathrm{P}_{\mathrm{SC}}^{s, k}\right)$ in $(21)$ only imposes that at most one user in one transmit direction can be selected at SC $k$ in frame $s$. Therefore, as the objective function in (21) is separable among users and transmit directions, the optimal solution to subproblem $\left(\mathrm{P}_{\mathrm{SC}}^{s, k}\right)$ in (21) (even if nonconvex) is either to allocate 0 power to all users and transmit directions associated to SC $k$ (i.e. $\mathbf{Q}_{i_{k}, s}^{d}=\mathbf{0}, \forall i_{k} \in \mathcal{I}_{k}, d$ ) or to allocate power only to the user and transmit direction that provides a larger value of its best contribution to the objective function in (21). As best contribution of a specific user in a specific transmit direction, we refer to the transmit covariance matrix that maximizes the objective function in (21) satisfying the associated inequality transmit power constraint. As the objective function is concave w.r.t. $\mathbf{Q}_{i_{k}, s}^{d}$ and the transmit power constraint is linear, a single best contribution exists. So, we can optimally solve subproblem $\left(\mathrm{P}_{\mathrm{SC}}^{s, k}\right)$ in $(21)$ for SC $k$ in frame $s$ through a two-step procedure:

- Step 1: compute all the individual best contributions of users and transmit directions associated to SC $k$ to the objective function in (21) (denoted by $\mathbf{Q}_{i_{k}, s}^{d \star}, \forall i_{k} \in \mathcal{I}_{k}, d$, in what follows).

- Step 2: select the individual best contribution that contributes more to the objective function in (21) (as imposed by the first constraint in (21)) and set the remaining transmit covariance matrices equal to $\mathbf{0}$. This corresponds to the optimal solution to subproblem $\left(\mathrm{P}_{\mathrm{SC}}^{s, k}\right)$ in $(21)$ and is denoted by $\mathbf{Q}_{i_{k}, s}^{d \star \star}$ in what follows.

1) Step 1: The individual best contribution of user $i_{k}$ in transmit direction $d$ to the objective function of subproblem $\left(\mathrm{P}_{\mathrm{SC}}^{s, k}\right)$ in $(21)$ is obtained by removing the first constraint in (21) and solving the associated optimization problem, i.e.:

$$
\begin{array}{cl}
\underset{\mathbf{Q}_{i_{k}, s}^{d} \succeq \mathbf{0}}{\operatorname{maximize}} & \mu_{i_{k}, s}^{d} \log _{2}\left|\mathbf{I}+\mathbf{H}_{i_{k}}^{d} \mathbf{Q}_{i_{k}, s}^{d} \mathbf{H}_{i_{k}}^{d H}\left(\mathbf{N}_{i_{k}, s}^{d}\right)^{-1}\right| \\
& -\operatorname{Tr}\left(\mathbf{Q}_{i_{k}, s}^{d} \mathbf{\Upsilon}_{i_{k}, s}^{d}\right)
\end{array}
$$

subject to $\operatorname{Tr}\left(\mathbf{Q}_{i_{k}, s}^{d}\right) \leq P^{\max }$ 
where, for the shake of presentation, we have unified parts of DL and UL nomenclature as:

$$
\mathbf{H}_{i_{k}}^{d}=\left\{\begin{array}{l}
\mathbf{H}_{k, i_{k}} \text { if } d=\mathrm{DL} \\
\mathbf{H}_{i_{k}, k} \text { if } d=\mathrm{UL}
\end{array}, P^{\max }=\left\{\begin{array}{l}
P_{\mathrm{SC}}^{\max } \text { if } d=\mathrm{DL} \\
P_{\mathrm{UE}}^{\max } \text { if } d=\mathrm{UL}
\end{array} .\right.\right.
$$

Problem in (22) is a convex optimization problem w.r.t. $\mathbf{Q}_{i_{k}, s}^{d}$ and thus it can be solved by the standard Lagrange duality method [28]. The result is included in Proposition 1 (and particularized for SISO systems afterwards).

Proposition 1: At SC $k$, the best contribution of user $i_{k}$ in transmit direction $d$ to the objective function of problem in (21) (i.e. the optimal solution for the transmit covariance matrix $\mathbf{Q}_{i_{k}, s}^{d \star}$ in case user $i_{k}$ in transmit direction $d$ was selected at SC $k$ in frame $s$ ) is given by:

$$
\mathbf{Q}_{i_{k}, s}^{d \star}=\left(\mathbf{B}_{i_{k}, s}^{d}\right)^{-\frac{H}{2}} \mathbf{V}_{i_{k}, s}^{d} \boldsymbol{\Sigma}_{i_{k}, s}^{d}\left(\mathbf{V}_{i_{k}, s}^{d}\right)^{H}\left(\mathbf{B}_{i_{k}, s}^{d}\right)^{-\frac{1}{2}},
$$

where $\Sigma_{i_{k}, s}^{d}=\operatorname{diag}\left(\sigma_{i_{k}, s}^{d}(1), \ldots, \sigma_{i_{k}, s}^{d}(\tilde{M})\right)$ is an $\tilde{M} \times \tilde{M}$ diagonal matrix $(\tilde{M}=M$ if $d=\mathrm{DL}$ or $\tilde{M}=N$ if $d=\mathrm{UL})$, and

$\mathbf{B}_{i_{k}, s}^{d}=\boldsymbol{\Upsilon}_{i_{k}, s}^{d}+\lambda_{i_{k}, s}^{d} \mathbf{I}$,

$\mathbf{V}_{i_{k}, s}^{d} \mathbf{Z}_{i_{k}, s}^{d} \mathbf{V}_{i_{k}, s}^{d H}=\left(\mathbf{B}_{i_{k}, s}^{d}\right)^{-\frac{1}{2}} \mathbf{H}_{i_{k}}^{d H}\left(\mathbf{N}_{i_{k}, s}^{d}\right)^{-1} \mathbf{H}_{i_{k}}^{d}\left(\mathbf{B}_{i_{k}, s}^{d}\right)^{-\frac{H}{2}}$,

$\sigma_{i_{k}, s}^{d}(n)=\left(\frac{\mu_{i_{k}, s}^{d}}{\ln (2)}-\frac{1}{z_{i_{k}, s}^{d}(n)}\right)^{+}, n=1, \ldots, \tilde{M}$,

being $\lambda_{i_{k}, s}^{d} \geq 0$ the Lagrange multiplier associated to the inequality transmit power constraint in (22). (26) denotes the eigenvalue decomposition of the matrix in the righthand side, where $\mathbf{V}_{i_{k}, s}^{d} \in \mathbb{C}^{\tilde{M} \times \tilde{M}}$ is a unitary matrix and $\mathbf{Z}_{i_{k}, s}^{d}=\operatorname{diag}\left(z_{i_{k}, s}^{d}(1), \ldots, z_{i_{k}, s}^{d}(\tilde{M})\right)$ is a diagonal matrix.

Proof: See Appendix B.

For general MIMO systems the solution in (24) depends on a single parameter: $\lambda_{i_{k}, s}^{d}$, which has to be optimized to meet the inequality transmit power constraint in (22) and has to satisfy complementary slackness, i.e. $\lambda_{i_{k}, s}^{d}\left(\operatorname{Tr}\left(\mathbf{Q}_{i_{k}, s}^{d}\right)-P^{\max }\right)=0$. $\lambda_{i_{k}, s}^{d}=0$ in case the power constraint is met with strict inequality, while $\lambda_{i_{k}, s}^{d}>0$ in case the power constraint is met with equality. The optimal value for $\lambda_{i_{k}, s}^{d}$ can be efficiently obtained using, for instance, a simple bisection method [28].

For SISO systems (i.e. $M=N=1$ ), in which all parameters are complex scalars, the solution in (24) can be obtained in closed-form. It is derived from Proposition 1 in what follows and the obtained solution corresponds to the distributed solution for power allocation in SISO interference channels that can be derived from [29]. Let $H_{i_{k}}^{\mathrm{DL}}=H_{k, i_{k}}$ and $H_{i_{k}}^{\mathrm{UL}}=H_{i_{k}, k}$ denote the DL and UL complex channels, respectively, see (23). Similarly, let $N_{i_{k}, s}^{\mathrm{DL}}$ and $N_{i_{k}, s}^{\mathrm{UL}}$ denote the interference-plusnoise received power in DL and UL, respectively, as shown in (9)-(10), and let $\Upsilon_{i_{k}, s}^{\mathrm{DL}}$ and $\Upsilon_{i_{k}, s}^{\mathrm{UL}}$ denote the cost in DL and UL, respectively, see (17)-(18). The optimization variables are the transmit power in $\mathrm{DL}\left(Q_{i_{k}, s}^{\mathrm{DL}}\right)$ and in $\mathrm{UL}\left(Q_{i_{k}, s}^{\mathrm{UL}}\right)$ instead of the transmit covariance matrices that appear in the MIMO case (see (3)-(4)). In the SISO case, the design of the Lagrange multiplier in (24) can be omitted because the constraint of the maximum transmit power can be directly imposed over the optimization variables: $Q_{i_{k}, s}^{d} \leq P^{\max }$, see (22). So, ignore $\lambda_{i_{k}, s}^{d}$ in (25), such that: $B_{i_{k}, s}^{d}=\Upsilon_{i_{k}, s}^{d}$. The EVD in (26) does not need to be performed in the SISO case, and we should simply set: $V_{i_{k}, s}^{d}=1$ and $Z_{i_{k}, s}^{d}=\left|H_{i_{k}}^{d}\right|^{2} /\left(\Upsilon_{i_{k}, s}^{d} N_{i_{k}, s}^{d}\right)$. This way, substituting said values into (27), we get:

$$
\sigma_{i_{k}, s}^{d}=\left(\frac{\mu_{i_{k}, s}^{d}}{\ln (2)}-\frac{\Upsilon_{i_{k}, s}^{d} N_{i_{k}, s}^{d}}{\left|H_{i_{k}}^{d}\right|^{2}}\right)^{+},
$$

and finally from (24):

$$
Q_{i_{k}, s}^{d}=\frac{\sigma_{i_{k}, s}^{d}}{\Upsilon_{i_{k}, s}^{d}}=\left(\frac{\mu_{i_{k}, s}^{d}}{\ln (2) \Upsilon_{i_{k}, s}^{d}}-\frac{N_{i_{k}, s}^{d}}{\left|H_{i_{k}}^{d}\right|^{2}}\right)^{+} .
$$

Therefore, for SISO systems, the best contribution of user $i_{k}$ in transmit direction $d$ to the objective function of problem in (21) (i.e. the optimal solution for the transmit power $Q_{i_{k}, s}^{d \star}$ in case user $i_{k}$ in transmit direction $d$ was selected at $\mathrm{SC} k$ in frame $s$ ) is directly obtained by constraining the value in (29) between 0 and the maximum available power $\left(P^{\max }\right)$, i.e.:

$$
Q_{i_{k}, s}^{d \star}=\min \left(\left(\frac{\mu_{i_{k}, s}^{d}}{\ln (2) \Upsilon_{i_{k}, s}^{d}}-\frac{N_{i_{k}, s}^{d}}{\left|H_{i_{k}}^{d}\right|^{2}}\right)^{+}, P^{\max }\right) .
$$

The SISO case allows gaining more insights on the obtained solution in (30). If the cost $\Upsilon_{i_{k}, s}^{d}$ is large (i.e. selecting user $i_{k}$ in transmit direction $d$ has a detrimental impact in terms of achievable rate over neighboring SCs/users), then the associated variable $Q_{i_{k}, s}^{d \star}$ tends to 0 . On the contrary, if the cost $\Upsilon_{i_{k}, s}^{d}$ tends to 0 (i.e. selecting user $i_{k}$ in transmit direction $d$ has not a detrimental impact over the achievable rate of neighboring SCs/users) then the associated variable $Q_{i_{k}, s}^{d \star}$ is given by the maximum power $\left(P_{\mathrm{SC}}^{\max }\right.$ or $\left.P_{\mathrm{UE}}^{\max }\right)$.

2) Step 2: Once the individual best contributions of all user and transmit directions at SC $k$ have been obtained (i.e. $\mathbf{Q}_{i_{k}, s}^{d \star}, \forall i_{k} \in \mathcal{I}_{k}, d$ ), since at most one user in a single transmit direction can be scheduled per-SC at every frame, the optimal solution to subproblem $\left(\mathrm{P}_{\mathrm{SC}}^{s, k}\right)$ in (21) corresponds either to select user $i_{k} \in \mathcal{I}_{k}$ and transmit direction $d \in\{\mathrm{DL}, \mathrm{UL}\}$ at $\mathrm{SC} k$ in frame $s$ providing a larger value of $\mu_{i_{k}, s}^{d} r_{i_{k}, s}^{d}\left(\mathbf{Q}_{i_{k}, s}^{d \star}\right)-\operatorname{Tr}\left(\mathbf{Q}_{i_{k}, s}^{d \star} \mathbf{\Upsilon}_{i_{k}, s}^{d}\right)$ if the largest value of $\mu_{i_{k}, s}^{d} r_{i_{k}, s}^{d}\left(\mathbf{Q}_{i_{k}, s}^{d \star}\right)-\operatorname{Tr}\left(\mathbf{Q}_{i_{k}, s}^{d \star} \mathbf{\Upsilon}_{i_{k}, s}^{d}\right)$ is positive or to select none (any user in any transmit direction) in case that the largest value of $\mu_{i_{k}, s}^{d} r_{i_{k}, s}^{d}\left(\mathbf{Q}_{i_{k}, s}^{d \star}\right)-\operatorname{Tr}\left(\mathbf{Q}_{i_{k}, s}^{d \star} \mathbf{\Upsilon}_{i_{k}, s}^{d}\right)$ is lower or equal to 0 (see objective function of subproblem $\left(\mathrm{P}_{\mathrm{SC}}^{s, k}\right)$ in $(21)$ ). This way, the optimal solution to subproblem $\left(\mathrm{P}_{\mathrm{SC}}^{s, k}\right)$ in (21) for SC $k$ in frame $s$, i.e. $\mathbf{Q}_{i_{k}, s}^{d \star \star}, \forall i_{k} \in \mathcal{I}_{k}, d$, is obtained.

The two-step procedure is summarized in Algorithm 1. For SC $k$, we have to check all contributions of its associated users (i.e. search on the set $\mathcal{I}_{k}$ ) in two transmit directions (i.e. DL and UL), which renders a search among $2\left|\mathcal{I}_{k}\right|$ possible values. But, let us recall that in SCNs the expected number of users that are associated to each SC is much lower than in conventional macrocell-based networks (i.e. low $\left|\mathcal{I}_{k}\right|$ ). Therefore, the complexity associated to Algorithm 1 is limited and, more important, it is not scaled with the network density as the number of users per SC is reduced with the SC density.

Remark 1: Algorithm 1 provides the global optimum solution to subproblem $\left(\mathrm{P}_{\mathrm{SC}}^{s, k}\right)$ in $(21)$ for SC $k$ in frame $s$. 


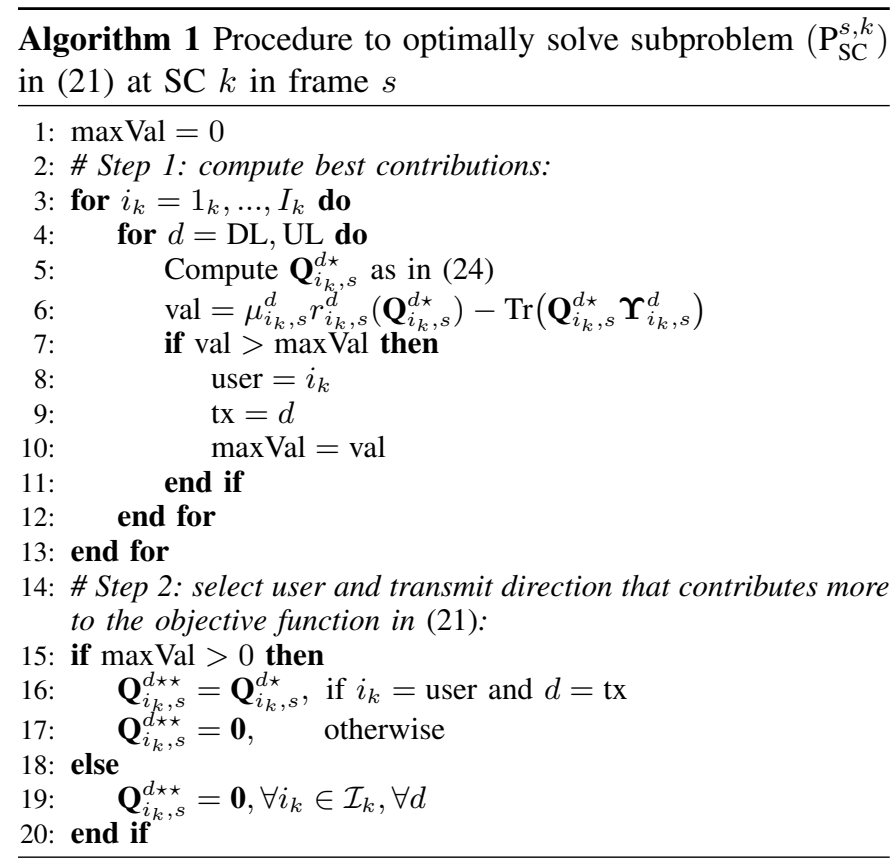

\section{Algorithm to solve (15)}

The overall algorithm to solve problem $\left(\mathrm{P}_{\text {frame }}^{s}\right)$ in (15) essentially solves subproblems $\left(\mathrm{P}_{\mathrm{SC}}^{s, k}\right)$ in $(21)$ iteratively until convergence. To that end, the Gauss-Seidel iteration (sequential optimizations), the Jacobi iteration (sequential optimizations), or an entirely asynchronous iteration (simultaneous optimizations) could be adopted, see [30].

Algorithm 2 details the procedure to solve problem $\left(\mathrm{P}_{\text {frame }}^{s}\right)$ in (15) in frame $s$ by following a Gauss-Seidel iteration. It starts from an initialization of the transmit covariance matrices $\left\{\mathbf{Q}_{i_{k}, s}^{d}\right\}$ that meets constraints in (15) (line 1). A suitable initialization is to select (for each SC $k$ ) the user $\left(i_{k}^{\star}\right)$ and transmit direction $\left(d^{\star}\right)$ with largest $\mu_{i_{k}, s}^{d} r_{i_{k}, s}^{d}$ (no cost) when using the achievable rates in (7)-(8) as a function of the useful signal power (no interference), maximum power, and precoding matrices that diagonalize the equivalent channel. After that, the iterative algorithm is performed in which subproblems $\left(\mathrm{P}_{\mathrm{SC}}^{s, k}\right)$ in $(21)$ are solved sequentially for all SCs. For each SC $k: a$ ) the interference-cost matrices in (17)(18) and the interference-plus-noise covariance matrices in (9)-(10) are computed (lines 5-6), and $b$ ) the optimization in Algorithm 1 is performed to obtain the transmit covariances matrices (including user scheduling, precoding design, and transmit direction selection) for SC $k$, i.e. $\left\{\mathbf{Q}_{i_{k}, s}^{d \star \star}\right\}$ (line 8). The procedure is iterated until convergence is reached and provides $\left\{\mathbf{Q}_{i_{k}, s}^{d \star \star}\right\}$ as output.

Proposition 2: Algorithm 2 converges to a limit point satisfying the Karush-Kuhn-Tucker (KKT) conditions of problem $\left(\mathrm{P}_{\text {frame }}^{s}\right)$ in (15).

Proof: See Appendix C.

Convergence of Algorithm 2 when the Gauss-Seidel iteration is applied can be proved by following similar steps as in [31] since the objective function of problem $\left(\mathrm{P}_{\text {frame }}^{s}\right)$ in (15) is not decreased at every iteration when sequential updates are performed and, in addition, the objective

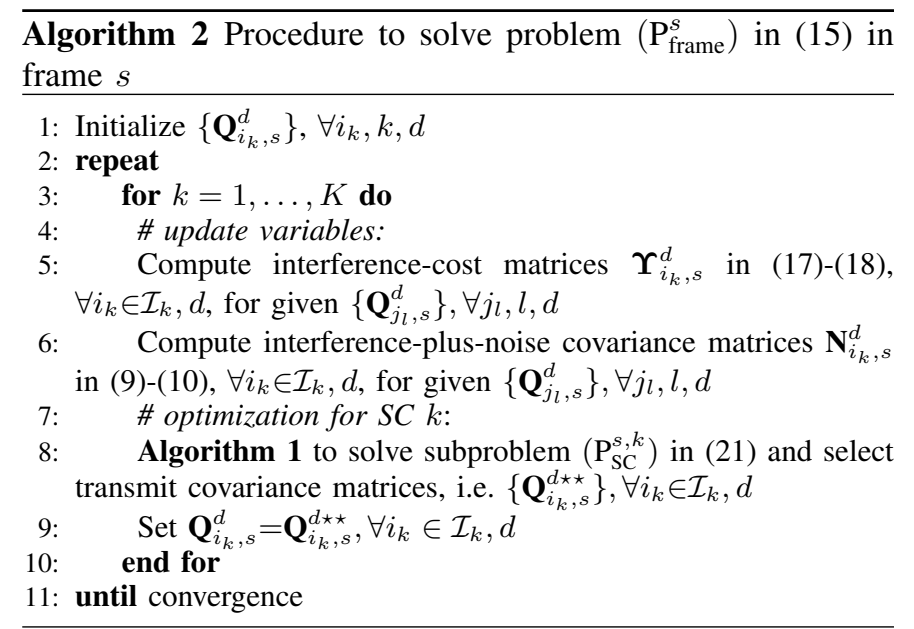

function is bounded from the above. Recall that with an entirely asynchronous iteration (simultaneous optimizations) convergence could not be guaranteed because the objective function of problem $\left(\mathrm{P}_{\text {frame }}^{s}\right)$ in (15) could oscillate when the interference-cost matrices were updated. In that case, some works have shown through simulations that convergence is achieved by performing simultaneous optimizations provided that a memory is included in the interference-cost matrices (as, for instance, through a low pass filter), see [25], [32].

Remark 2: Algorithm 2 is executed at the cluster controller, which disposes of CSI of all links in the cluster. Alternatively, it could be performed in a distributed manner at each SC provided that CSI was available at each SC and information exchange was used iteratively among SCs to get the interference-cost matrices $\Upsilon_{i_{k}, s}^{d}$ in (17)-(18). But, due to the short-length of the frame, distributed implementation might not be feasible as it would entail longer delays.

Remark 3: The proposed Algorithm 2 can be used to determine the user scheduling, precoding design, and power control even if the transmit direction per SC is fixed in frame $s$, simply by not optimizing the transmit covariance matrices $\mathbf{Q}_{i_{k}, s}^{d}$ of transmit directions not allowed at SCs.

Remark 4: For SISO systems, the proposed Algorithm 2 can be adopted to determine the user scheduling and transmit direction selection when binary power control is adopted. In this case, the computation of the optimal values for the power allocation in (30) is not needed. We should simply replace the optimization rule in line 5 of Algorithm 1 by $Q_{i_{k}, s}^{\mathrm{DL} \star}=P_{\mathrm{SC}}^{\max }$ and $Q_{i_{k}, s}^{\mathrm{UL}}=P_{\mathrm{UE}}^{\max }$.

Remark 5: Under Orthogonal Frequency Division Multiple Access (OFDMA) ${ }^{3}$ with a power spectral mask, where users for the same SC are assumed to be orthogonal in frequency domain and the primary source of interference is inter-cell interference, the proposed Algorithm 2 can still be used to determine the user scheduling and precoding design on each frequency subband with a slight modification: we should impose that the same transmit direction is adopted for the

${ }^{3}$ OFDMA is the radio access technology employed in downlink transmission of 3GPP LTE and LTE-A systems [33], as well as in both uplink and downlink of IEEE $802.16 \mathrm{~m}$ advanced WiMAX [34]. Furthermore, it is one of the major candidates for different use cases of future 5G systems [35]. 


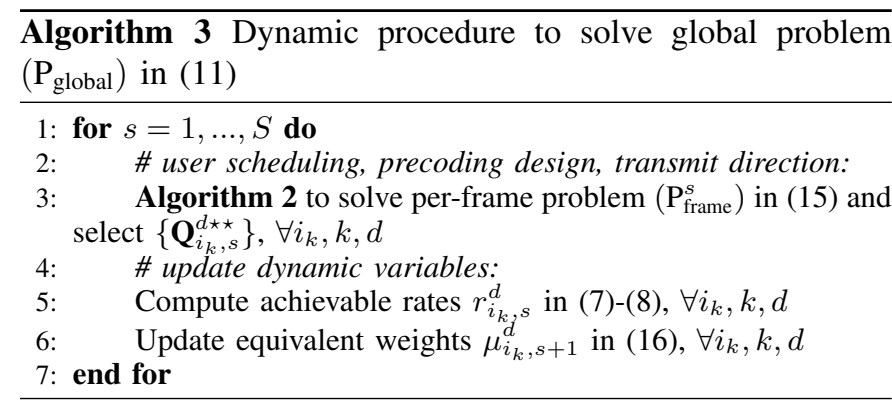

whole set of subbands on a given frame $s$ at every SC. Therefore, each SC should check all subbands in DL and all subbands in UL and then chose the best transmit direction with the associated user scheduling and transmit precoding. When a power spectral mask is not included, then the solution is not straightforward due to the transmit power constraints that couple different subbands.

Remark 6: To reduce the complexity of the proposed algorithm, one could derive a new approach based on a sequential optimization in which decisions of the transmit direction and scheduling/precoding for each frame were decoupled. The transmit direction selection could be done by following a proportional fair criterion at each frame (either coordinated or not among SCs). Afterwards, the proposed algorithm could be applied to determine the user scheduling and transmit precoding at each frame. However, this lies out of the scope of the paper, as we have focused on the joint optimization of all the resource allocation variables.

\section{Algorithm to solve (11)}

Finally, for completion, Algorithm 3 includes the dynamic user scheduling, precoding design, and transmit direction selection to solve the global problem $\left(\mathrm{P}_{\text {global }}\right)$ in (11) through an on-line solution. At every frame $s$, Algorithm 2 is executed to design the final $\left\{\mathbf{Q}_{i_{k}, s}^{d \star \star}\right\}$ (line 3) and, then, the weights $\mu_{i_{k}, s+1}^{d}$ to be used in the subsequent frame $s+1$ are updated (line 6).

\section{Simulation Results}

The simulation scenario consists of a synchronized TDD deployment of $K$ outdoor SCs, which are randomly placed within a circular area of $100 \mathrm{~m}$ radius with a minimum distance of $40 \mathrm{~m}$ among them. The $K$ SCs are managed by a cluster controller. $I_{k}$ users are randomly placed around each SC $k$ in a concentric $40 \mathrm{~m}$ radius circle. The evaluation methodology (including system parameters, propagation characteristics, and traffic modeling) designed by 3GPP for outdoor pico scenario in [5] is followed and summarized in Table I. We focus on the evaluation in a single subband adopting a narrow-band channel model.

For simulation purposes the same traffic asymmetry $\left(a_{i_{k}}\right)$ is used for all users, $\forall i_{k}, \forall k$. Different values of the traffic asymmetries $\left(a_{i_{k}}\right)$, network densities $(K)$, and user densities $\left(I_{k}\right)$ are used for simulations. $S=100$ frames are used. Different MIMO antenna configurations are evaluated: $M=N=1$,
TABLE I: Simulation Assumptions.

\begin{tabular}{|c|c|}
\hline \multicolumn{2}{|c|}{ General system parameters } \\
\hline SCs deployment & $\begin{array}{l}K \text { SCs, randomly deployed, circular area } 100 \\
\text { m radius }\end{array}$ \\
\hline Users deployment & $\begin{array}{l}I_{k} \text { users per SC, randomly deployed, concentric } \\
\text { circular area } 40 \mathrm{~m} \text { radius around the } k \text {-th SC }\end{array}$ \\
\hline Carrier frequency & $2 \mathrm{GHz}$ \\
\hline SC transmit power & $24 \mathrm{dBm}$ \\
\hline UE transmit power & $23 \mathrm{dBm}$ \\
\hline SC antenna system & $5 \mathrm{dBi}, 2 \mathrm{D}$, Omnidirectional, $M$ antennas \\
\hline UE antenna system & $0 \mathrm{dBi}, 2 \mathrm{D}$, Omnidirectional, $N$ antennas \\
\hline Noise figure & $9 \mathrm{~dB}$ \\
\hline Noise power & $-84 \mathrm{dBm}$ \\
\hline \multicolumn{2}{|r|}{ Propagation characteristics } \\
\hline $\begin{array}{l}\text { Pathloss SC-to-SC } \\
(d \text { in } \mathrm{Km})\end{array}$ & $\begin{array}{l}\text { LOS: PL }=98.4+20 \log _{10}(d) \mathrm{dB} \\
\text { NLOS: } \mathrm{PL}=169.36+40 \log _{10}(d) \mathrm{dB}\end{array}$ \\
\hline $\begin{array}{l}\text { Pathloss SC-to-UE } \\
(d \text { in } \mathrm{Km})\end{array}$ & $\begin{array}{l}\text { LOS: } \mathrm{PL}=103.8+20.9 \log _{10}(d) \mathrm{dB} \\
\text { NLOS: } \mathrm{PL}=145.4+37.5 \log _{10}(d) \mathrm{dB}\end{array}$ \\
\hline $\begin{array}{l}\text { Pathloss UE-to-UE } \\
(d \text { in } \mathrm{Km})\end{array}$ & $\begin{array}{l}d<0.05: \mathrm{PL}=98.45+20 \log _{10}(d) \mathrm{dB} \\
d \geq 0.05: \mathrm{PL}=175.78+40 \log _{10}(d) \mathrm{dB}\end{array}$ \\
\hline LOS probability & $\begin{array}{l}0.5-\min (0.5,5 \exp (-0.156 / d)) \\
+\min (0.5,5 \exp (-d / 0.03))\end{array}$ \\
\hline Shadowing SC-to-SC & $6 \mathrm{~dB}$ \\
\hline Shadowing SC-to-UE & LOS: $3 \mathrm{~dB}$, NLOS: $4 \mathrm{~dB}$ \\
\hline Shadowing UE-to-UE & $6 \mathrm{~dB}$ \\
\hline \multicolumn{2}{|r|}{ Other parameters } \\
\hline $\begin{array}{l}\text { Traffic model } \\
\text { Link adaptation }\end{array}$ & $\begin{array}{l}\text { Full-load } \\
\text { Ideal }\end{array}$ \\
\hline \multicolumn{2}{|r|}{ Optimization time scales } \\
\hline 'CS-CP-CD' & $\begin{array}{l}\text { user scheduling, precoding: } 1 \text { frame, } \\
\text { transmit direction: } 1 \text { frame }\end{array}$ \\
\hline 'CS-CP-uD' & $\begin{array}{l}\text { user scheduling, precoding: } 1 \text { frame, } \\
\text { transmit direction: } 10 \text { frames }\end{array}$ \\
\hline 'uS-uP-uD' & $\begin{array}{l}\text { user scheduling, precoding: } 1 \text { frame, } \\
\text { transmit direction: } 10 \text { frames }\end{array}$ \\
\hline
\end{tabular}

$M=N=2$, and $M=N=4$ (depicted in figures as $1 \times 1,2 \times 2$, and $4 \times 4$, respectively).

A proportional fair $(\mathrm{PF})$ criterion for problem $\left(\mathrm{P}_{\text {global }}\right)$ in (11) is adopted, i.e. $u(z)=\log (z)$. Therefore, the objective function of problem $\left(\mathrm{P}_{\text {global }}\right)$ in (11) corresponds to a weighted sum of the log of average rates:

$$
f_{\text {global }}=\sum_{\substack{k \in \mathcal{K} \\ i_{k} \in \mathcal{I}_{k}}}\left(a_{i_{k}} \log \left(\bar{R}_{i_{k}}^{\mathrm{DL}}\right)+\left(1-a_{i_{k}}\right) \log \left(\bar{R}_{i_{k}}^{\mathrm{UL}}\right)\right) .
$$

The aim of the present simulations is to show the benefits of jointly optimizing the transmit direction selection and the conventional allocation policies (including user scheduling and precoding design) through the use of the proposed Algorithm 2 as compared to schemes where the transmit direction is set at each SC according to the traffic asymmetries of the associated users while the conventional allocation policies are coordinated among SCs.

We compare the following schemes:

- CS-CP-CD: proposed scheme in this work where the user scheduling, precoding design, and transmit direction selection are dynamically optimized at every frame by following Algorithm 2 under a PF criterion. Thus, in addition to conventional coordinated scheduling and coordinated precoding (CS-CP), coordinated transmit direction 
(CD) is included.

- CS-CP-uD: SC-specific TDD scheme with coordinated scheduling and coordinated precoding (CS-CP) but uncoordinated transmit direction (uD) [5], [6]. In particular, 'CS-CP-uD' corresponds to the cell-specific dynamic DL/UL reconfiguration scheme detailed in $[6 \text {, Sect. II }]^{4}$. The transmit direction per SC is set according to the traffic asymmetries of the associated users (e.g. if $a_{i_{k}}=0.7$ then 7 frames are used for DL and the consecutive 3 frames are used for UL). Then, once the transmit direction has been set, the user scheduling and transmit precoding matrices are optimized at every frame under a PF criterion to maximize the weighted sum-rate of the system (instead of following the power leakage minimization proposed in [6]) for a fair comparison with 'CS-CP-CD'.

- uS-uP-uD: SC-specific TDD scheme without coordination. The transmit direction per SC is set according to the traffic asymmetries of the associated users (similar to 'CS-CP-uD'). The user scheduling and transmit precoding are selected at each SC independently (in a selfish manner at every frame) by following a PF criterion.

Therefore, for 'CS-CP-CD', joint decisions on the transmit direction, user scheduling, and precoding are taken on a frame basis by following PF criterion (coordinated among SCs). Differently, for 'CS-CP-uD' and 'uS-uP-uD', decisions on the transmit direction are taken on a 10-frame basis based on the traffic asymmetries while joint decisions on the user scheduling and precoding are taken on a frame basis by following $\mathrm{PF}$ criterion (coordinated for 'CS-CP-uD' and uncoordinated for 'uS-uP-uD'). Table I details the optimization time scales.

As stated in the introduction, SC-specific decisions combined with interference coordination techniques (i.e. 'CS-CPuD' described above) achieve the best performance among existing dynamic TDD schemes [6], [10]. Also note that as the same traffic asymmetry $\left(a_{i_{k}}\right)$ is considered for all users in the simulation setup, then all SCs would use the same DLUL pattern under 'CS-CP-uD' and 'uS-uP-uD' schemes (but possibly not with 'CS-CP-CD').

Simulations will allow us to identify the gains that are obtained from CS-CP when the transmit direction is fixed to meet the traffic asymmetries (i.e. 'CS-CP-uD' versus 'uS-uP-uD'), and to determine in which situations the joint optimization of the transmit direction together with CS-CP provides additional gains (i.e. 'CS-CP-CD' versus 'CS-CP-uD').

\section{A. Convergence of Algorithm 2}

First, we demonstrate convergence of the proposed Algorithm 2, which solves the per-frame problem $\left(\mathrm{P}_{\text {frame }}^{s}\right)$ in (15). Results corresponds to 'CS-CP-CD' scheme for a specific frame and a concrete deployment. Fig. 4 shows the evolution of the weighted sum-rate for $K=6, I_{k}=2$, and different antenna configurations $(1 \times 1,2 \times 2$, and $4 \times 4)$. For this specific

\footnotetext{
${ }^{4}$ In [6], the transmit directions are constrained to the LTE frame patterns and are selected for the entire LTE frame that is composed of 10 LTE subframes. However, as the minimum time interval after which the transmit direction can be changed in LTE is the LTE subframe, we associate the LTE subframe with the frame in Fig. 1 to simulate the algorithm in [6] when the suggested 5G frame structure is adopted.
}

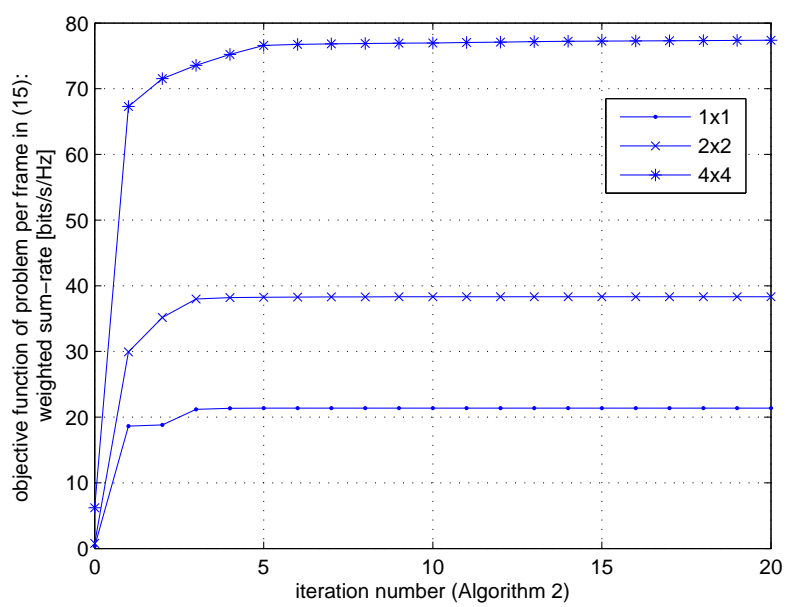

Fig. 4: Convergence of Algorithm 2. Weighted sum-rate (objective function of problem $\left(\mathrm{P}_{\text {frame }}^{s}\right)$ in (15)) versus iteration number. $K=6, I_{k}=2, \mu_{i_{k}, s}^{d}=0.5$.

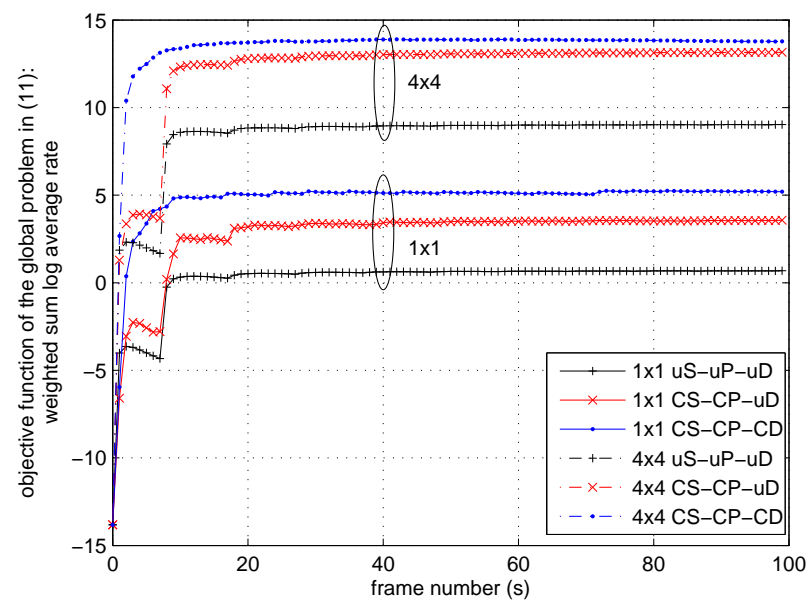

Fig. 5: Evolution of Algorithm 3. Weighted sum of the log average rate (objective function $f_{\text {global }}$ in (31)) versus frame number. $K=6, I_{k}=1, a_{i_{k}}=0.7, S=100$.

evaluation, the weighting coefficients for $\left(\mathrm{P}_{\text {frame }}^{s}\right)$ in (15) are set to $\mu_{i_{k}, s}^{d}=0.5, \forall i_{k}, k, d$. It can be observed that the weighted sum-rate converges quite fast. Also, the monotonic increase of the weighted sum-rate is verified.

\section{B. Evolution of on-line solutions (Algorithm 3)}

Second, let us show the time evolution of the proposed Algorithm 3, which solves the global problem ( $\left.\mathrm{P}_{\text {global }}\right)$ in (11) for a concrete deployment. Fig. 5 displays the evolution of the global objective function $f_{\text {global }}$ in (31) versus the frame number (i.e. $s=1, \ldots, S$ ) for $K=6, I_{k}=1, a_{i_{k}}=0.7$, and two different antenna configurations $(1 \times 1$ and $4 \times 4)$. It is shown that the weighted sum of the log average rates increases with the frame number and then gets stabilized for all schemes, such that on-line solutions converge. The values in which each scheme gets stabilized will be compared in next sections through simulation results averaged over different deployments. 


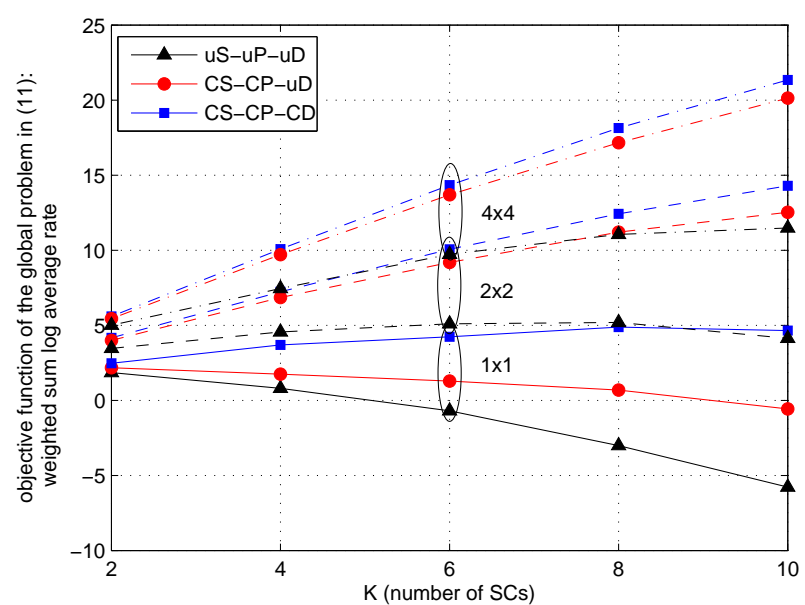

Fig. 6: Mean weighted sum of the log average rate (objective function $f_{\text {global }}$ in (31)) versus number of SCs $(K) . I_{k}=1, a_{i_{k}}=0.7, S=100$.

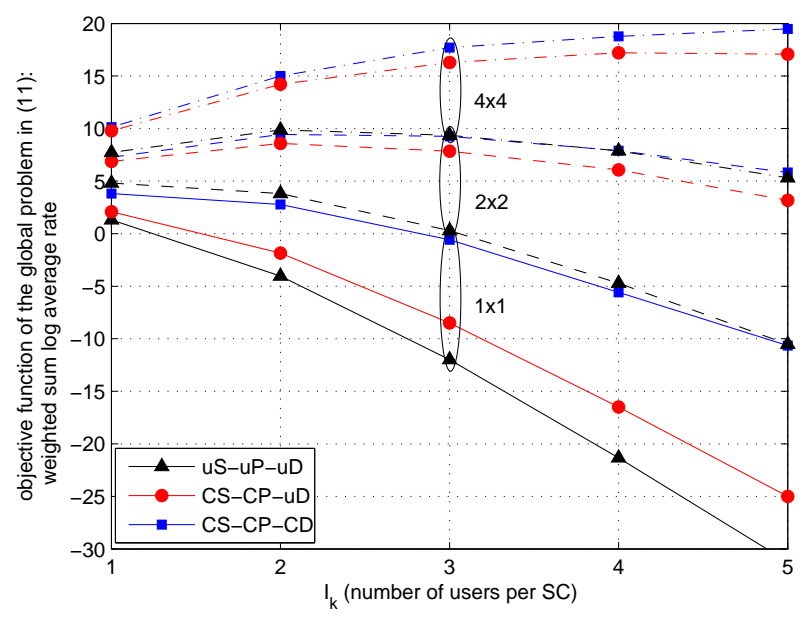

Fig. 7: Mean weighted sum of the $\log$ average rate (objective function $f_{\text {global }}$ in (31)) versus number of users per SC $\left(I_{k}\right) . K=4, a_{i_{k}}=0.7, S=100$.

\section{Results of the global utility for different network/user} densities and traffic asymmetries

In this section we show results averaged over 500 different random deployments, for $S=100$ and different antenna configurations $(1 \times 1,2 \times 2$, and $4 \times 4)$. The figures depict the mean of the objective function of the global problem $\left(\mathrm{P}_{\text {global }}\right)$ in (11) (i.e. $f_{\text {global }}$ in (31)) versus different system parameters. The $\mathrm{X}$-axis in Fig. 6 corresponds to the number of SCs within the cluster $(K)$ and the considered system setup is $I_{k}=1, a_{i_{k}}=0.7$. In Fig. 7, results are displayed as a function of the number of users per SC $\left(I_{k}\right)$ for $K=4, a_{i_{k}}=0.7$. Finally, Fig. 8 shows the objective function versus the traffic asymmetry $\left(a_{i_{k}}\right)$ for $K=4, I_{k}=1$.

We can observe in Figures 6-7-8 that the proposed ' $\mathrm{CS}-\mathrm{CP}$ CD' outperforms 'CS-CP-uD' for all antenna configurations, traffic asymmetries, network densities, and user densities. This is thanks to the optimization of the transmit direction selection jointly with the conventional allocation policies. The gains of 'CS-CP-CD' as compared to 'CS-CP-uD' are larger for $1 \times 1$

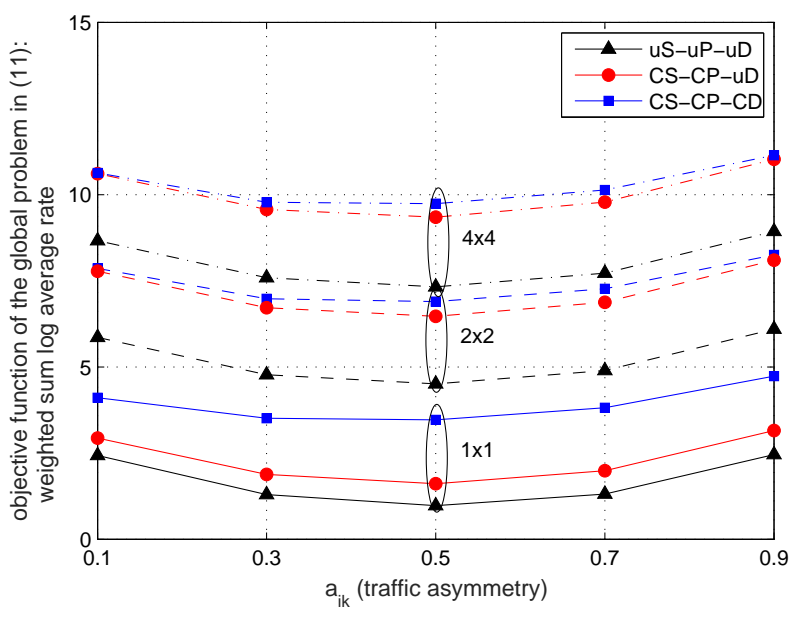

Fig. 8: Mean weighted sum of the $\log$ average rate (objective function $f_{\text {global }}$ in (31)) versus traffic asymmetry $\left(a_{i_{k}}\right) . K=4, I_{k}=1, S=100$.

than for $2 \times 2$ and $4 \times 4$ because when increasing the number of antennas more interference can be already managed through CS-CP and thus 'CS-CP-uD' does already provide significant gains over 'uS-uP-uD'. The gains are not much dependent on the traffic asymmetry condition (see Fig. 8) but, however, they do vary with the network density and the user density.

As the network density increases (i.e. as $K$ increases, see Fig. 6), significant gains are obtained with the coordinated strategies ('CS-CP-uD' and 'CS-CP-CD') because more interference is present in the network and thus more important becomes the coordination of the transmit strategies in the network. In addition, the gains of the proposed 'CS-CP-uD' technique as compared to 'CS-CP-CD' also increase with the network density. So, the joint optimization of the transmit direction and the allocation policies becomes important for interference management in dense networks.

As the user density increases (i.e. as $I_{k}$ increases, see Fig. 7), large gains are obtained with the coordinated strategies ('CS-CP-uD' and 'CS-CP-CD') because there is more flexibility to manage interference (i.e. there are more users to schedule). The largest gain from 'CS-CP-CD' over 'CS-CPuD' appear however in the $1 \times 1$ antenna configuration, owing to the fact that in the $2 \times 2$ and $4 \times 4$ flexibility is already provided with the number of antennas and hence the gains of 'CS-CP-CD' over 'CS-CP-uD' are not as remarkable as those of 'CS-CP-uD' over 'uS-uP-uD'.

\section{Results of the average rate}

Figure 9 depicts the cumulative density function (CDF) of the average rates obtained in DL and in UL, separately, for $K=4, I_{k}=2, S=100, a_{i_{k}}=0.7$, and different antenna configurations: $1 \times 1,2 \times 2$, and $4 \times 4$ (on each subplot).

Table II summarizes the relative gains in the mean of the average rates of 'CS-CP-uD' over 'uS-uP-uD' (labeled as CS$\mathrm{CP}$ ) and of 'CS-CP-CD' over 'CS-CP-uD' (labeled as +CD) for the different antenna configurations.

As it is expected, for $a_{i_{k}}=0.7$ (i.e. DL traffic is larger than $\mathrm{UL}$ traffic), DL average rates are larger than UL average rates 


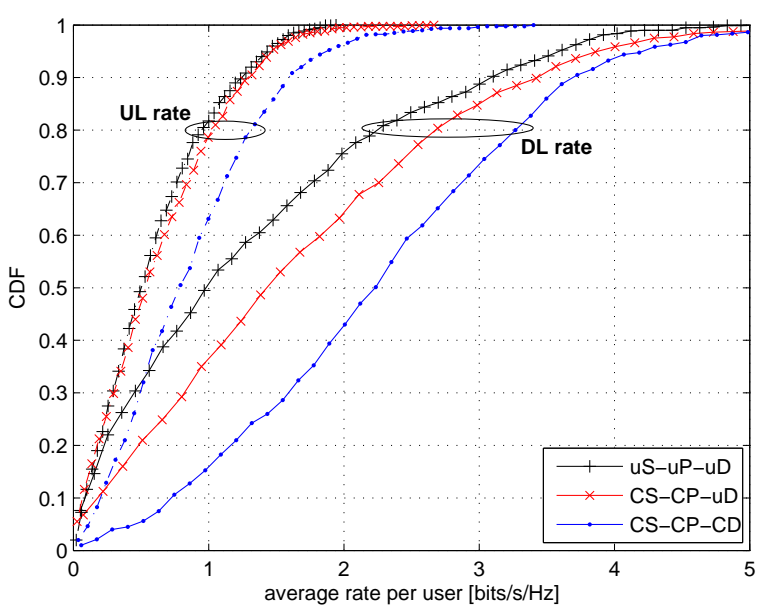

(a) $1 \times 1$

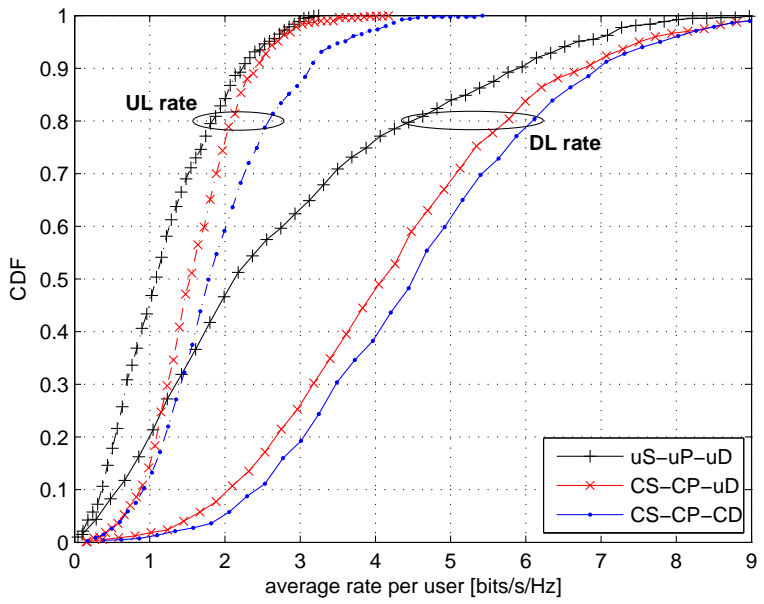

(b) $2 \times 2$

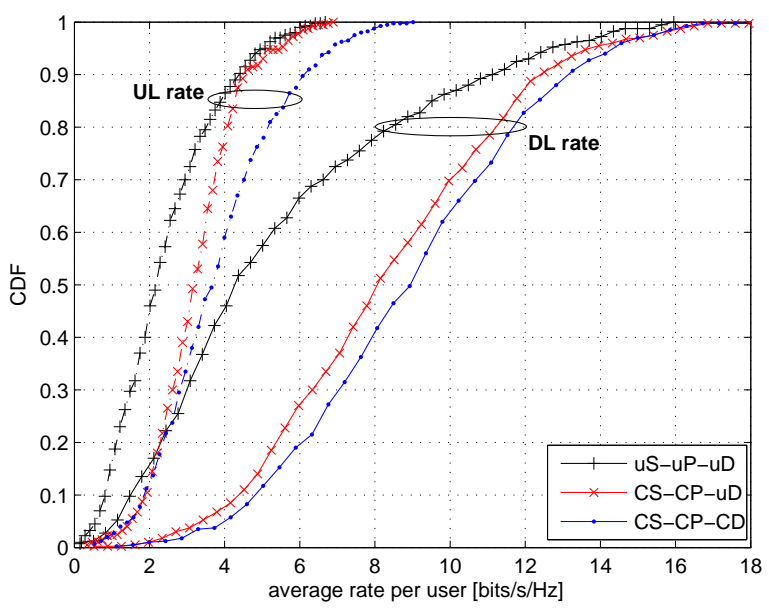

(c) $4 \times 4$

Fig. 9: CDF of the average rate (in bits/s/Hz) in DL and in UL. $K=4, I_{k}=2, S=100, a_{i_{k}}=0.7$.

(see Fig. 9). For $1 \times 1$ (see Fig. 9.(a)), 'CS-CP-CD' provides a significant improvement of both the outage and the mean of the average rate in DL and UL. The mean gains of 'CS-CP-CD' over 'CS-CP-uD' are 49-60\% (see Table II). For $2 \times 2$ and $4 \times 4$
TABLE II: RELATIVE GAINS OF 'CS-CP-UD' OVER 'US-UP-UD' AND OF 'CS-CP-CD' OVER 'CS-CP-UD' IN THE MEAN OF THE AVERAGE RATES IN DL AND UL, SEPARATELY. $K=4, I_{k}=2, S=100, a_{i_{k}}=0.7$.

\begin{tabular}{c||cc|cc|cc}
\cline { 2 - 7 } \multicolumn{1}{c||}{} & \multicolumn{2}{c|}{$1 \times 1$} & \multicolumn{2}{c|}{$2 \times 2$} & \multicolumn{2}{c}{$4 \times 4$} \\
\cline { 2 - 7 } & DL & UL & DL & UL & DL & UL \\
\hline \hline CS-CP & $46 \%$ & $7 \%$ & $95 \%$ & $45 \%$ & $86 \%$ & $42 \%$ \\
+ CD & $60 \%$ & $49 \%$ & $11 \%$ & $16 \%$ & $10 \%$ & $15 \%$ \\
\hline
\end{tabular}

cases (see Fig. 9.(b)-(c)), 'CS-CP-uD' does already provide an important enlargement of the outage rates and improves the mean average rate around $42-95 \%$ over 'uS-uP-uD' (see Table II), thus leaving less improvement for 'CS-CP-CD'. However, 'CS-CP-CD' allows still improving the rates in both transmit directions as compared to 'CS-CP-uD' and provides gains between $10-16 \%$ (see Table II). So the gains of 'CS-CP-CD' over 'CS-CP-uD' are reduced with the number of antennas, but not depreciable.

Let us remark that the gains of 'CS-CP-CD' over 'CS-CPuD' come from the proposed joint optimization. The suggested 5G frame structure in Fig. 1 allows applying the algorithm as it is. Even so, if there were $2^{10}$ LTE frame patterns available in LTE TDD, then we could have applied the algorithm to LTE without additional constraints and would get the benefits of the proposed joint optimization as well.

To conclude, from Figures 6-7-8-9, we infer that:

- larger gains are obtained for $1 \times 1$ than for $2 \times 2$ and $4 \times 4$, because the $1 \times 1$ setup has less flexibility for interference management and is therefore more benefited from the optimization of the transmit direction. Even though, positive gains are still reported when increasing the number of transmit/receive antennas.

- the gains of 'CS-CP-CD' increase as the network density increases (i.e. as $K$ increases), due to the fact that as $K$ increases then more interference is present and the optimization of the transmit direction becomes important for interference management.

- the gains of 'CS-CP-CD' increase as the user density increases (i.e. as $I_{k}$ increases), because by increasing $I_{k}$ more flexibility is available to select the proper user and transmit direction.

Consequently, the joint optimization of the transmit direction and the conventional allocation policies becomes key for interference management and it is specially important for interference-limited systems with a low number of antennas.

\section{CONCLUSIONS}

This paper presents a dynamic procedure for joint user scheduling, precoding design, and transmit direction selection in dynamic TDD MIMO small cell networks. Differently from previous works, the transmit direction is optimized at every frame jointly with the conventional allocation policies that include user scheduling, precoding design, and power control. Hence, a high adaptability to the instantaneous traffic and interference conditions in the network is achieved. Simulation results show gains in DL and UL average rates for different traffic asymmetries, network densities, and user densities as 
compared to existing schemes for dynamic TDD. The gains are larger for systems with a low number of antennas because they have less flexibility for interference management through the coordination of transmit/receive spatial filters and hence larger gains are obtained by optimizing the transmit direction.

Interesting future work includes the superposition coding transmission to multiple users served at every frame either in DL or in UL, whereby user grouping strategies should be optimized together with the precoding design and transmit direction selection. The application of the proposed approach to OFDMA-based networks without a power spectral mask is relevant as well. In this case, users from the same SC would be assigned to orthogonal resources at each frame, but each frame can be only devoted either for DL or UL and a single power constraint is to be included. Finally, a potential research line includes the reformulation of the problem in order to optimize the energy efficiency of the network subject to minimum per-user DL/UL average rate constraints such that quality of service requirements of the users are satisfied and traffic asymmetry conditions are met.

\section{ACKNOWLEDGMENTS}

This work has been supported by Huawei Technologies Co. Ltd. The authors would like to thank the anonymous reviewers for their constructive comments and valuable suggestions to improve the quality of the paper.

\section{APPENDIX A \\ DECOMPOSITION OF (15)}

In this section we show how the per-frame problem $\left(\mathrm{P}_{\text {frame }}^{s}\right)$ in (15) is decomposed into $K$ subproblems (one per SC), hence obtaining the subproblems $\left(\mathrm{P}_{\mathrm{SC}}^{s, k}\right)$ in $(21)(\forall k)$.

As the constraints of problem ( $\mathrm{P}_{\text {frame }}^{s}$ ) in (15) can be directly decomposed per SC, we focus on the objective function. To isolate the interference that renders the objective function of problem $\left(\mathrm{P}_{\text {frame }}^{s}\right)$ in (15) non-convex, define the weighted sumrate of the links different than those related to $\mathrm{SC} k$ as:

$$
f_{-k}\left(\mathbf{Q}_{k, s}, \mathbf{Q}_{-k, s}\right)=\sum_{l \neq k, j_{l} \in \mathcal{I}_{l}}\left(\mu_{j_{l}, s}^{\mathrm{DL}} r_{j_{l}, s}^{\mathrm{DL}}+\mu_{j_{l}, s}^{\mathrm{UL}} r_{j_{l}, s}^{\mathrm{UL}}\right),
$$

where $\mathbf{Q}_{k, s}$ refers to the set of transmit covariance matrices of all users and transmit directions related to SC $k$ and $\mathbf{Q}_{-k, s}$ denotes the set of transmit covariance matrices of all users and transmit directions not related to SC $k$, i.e. $\mathbf{Q}_{k, s} \triangleq\left\{\mathbf{Q}_{1_{k}, s}^{\mathrm{DL}}, \ldots, \mathbf{Q}_{I_{k}, s}^{\mathrm{DL}}, \mathbf{Q}_{1_{k}, s}^{\mathrm{UL}}, \ldots, \mathbf{Q}_{I_{k}, s}^{\mathrm{UL}}\right\}$ and $\mathbf{Q}_{-k, s} \triangleq\left\{\mathbf{Q}_{1, s}, \ldots, \mathbf{Q}_{k-1, s}, \mathbf{Q}_{k+1, s}, \ldots, \mathbf{Q}_{K, s}\right\}$. Using first order Taylor's expansion of $f_{-k}\left(\mathbf{Q}_{k, s}, \mathbf{Q}_{-k, s}\right)$ in (32) around $\overline{\mathbf{Q}}_{k, s}$ (i.e. $\overline{\mathbf{Q}}_{i_{k}, s}^{\mathrm{DL}}$ and $\overline{\mathbf{Q}}_{i_{k}, s}^{\mathrm{UL}}, \forall i_{k} \in \mathcal{I}_{k}$ ), we have:

$$
\begin{aligned}
& f_{-k}\left(\mathbf{Q}_{k, s}, \mathbf{Q}_{-k, s}\right) \approx f_{-k}\left(\overline{\mathbf{Q}}_{k, s}, \mathbf{Q}_{-k, s}\right) \\
& +\sum_{i_{k} \in \mathcal{I}_{k}} \operatorname{Tr}\left(\left.\left(\mathbf{Q}_{i_{k}, s}^{\mathrm{DL}}-\overline{\mathbf{Q}}_{i_{k}, s}^{\mathrm{DL}}\right) \frac{\delta f_{-k}\left(\mathbf{Q}_{k, s}, \mathbf{Q}_{-k, s}\right)}{\delta \mathbf{Q}_{i_{k}, s}^{\mathrm{DL}}}\right|_{\overline{\mathbf{Q}}_{i_{k}, s}^{\mathrm{DL}}}\right) \\
& +\sum_{i_{k} \in \mathcal{I}_{k}} \operatorname{Tr}\left(\left.\left(\mathbf{Q}_{i_{k}, s}^{\mathrm{UL}}-\overline{\mathbf{Q}}_{i_{k}, s}^{\mathrm{UL}}\right) \frac{\delta f_{-k}\left(\mathbf{Q}_{k, s}, \mathbf{Q}_{-k, s}\right)}{\delta \mathbf{Q}_{i_{k}, s}^{\mathrm{UL}}}\right|_{\overline{\mathbf{Q}}_{i_{k}, s}^{\mathrm{UL}}}\right) .
\end{aligned}
$$

The negative partial derivatives of $f_{-k}\left(\mathbf{Q}_{k, s}, \mathbf{Q}_{-k, s}\right)$ in (32) w.r.t. each matrix composing the set $\mathbf{Q}_{k, s}$ evaluated at $\overline{\mathbf{Q}}_{k, s}$ (i.e. $\overline{\mathbf{Q}}_{i_{k}, s}^{\mathrm{DL}}$ and $\overline{\mathbf{Q}}_{i_{k}, s}^{\mathrm{UL}}, \forall i_{k} \in \mathcal{I}_{k}$, see (33)) are given by:

$$
\begin{aligned}
\boldsymbol{\Upsilon}_{i_{k}, s}^{\mathrm{DL}} & =-\left.\left.\frac{\delta f_{-k}\left(\mathbf{Q}_{k, s}, \mathbf{Q}_{-k, s}\right)}{\delta \mathbf{Q}_{i_{k}, s}^{\mathrm{DL}}}\right|_{\overline{\mathbf{Q}}_{i_{k}, s}^{\mathrm{DL}}}\left(\mu_{j_{l}, s}^{\mathrm{DL}} \frac{\delta r_{j_{l}, s}^{\mathrm{DL}}}{\delta \mathbf{Q}_{i_{k}, s}^{\mathrm{DL}}}+\mu_{j_{l}, s}^{\mathrm{UL}} \frac{\delta r_{j_{l}, s}^{\mathrm{UL}}}{\delta \mathbf{Q}_{i_{k}, s}^{\mathrm{DL}}}\right)\right|_{\overline{\mathbf{Q}}_{i_{k}, s}^{\mathrm{DL}}}, \\
& =-\sum_{l \neq k, j_{l} \in \mathcal{I}_{l}} \delta \\
\mathbf{\Upsilon}_{i_{k}, s}^{\mathrm{UL}} & =-\left.\left.\frac{\delta f_{-k}\left(\mathbf{Q}_{k, s}, \mathbf{Q}_{-k, s}\right)}{\delta \mathbf{Q}_{i_{k}, s}^{\mathrm{UL}}}\right|_{\overline{\mathbf{Q}}_{i_{k}, s}^{\mathrm{UL}}}\left(\mu_{j_{l}, s}^{\mathrm{DL}} \frac{\delta r_{j_{l}, s}^{\mathrm{DL}}}{\delta \mathbf{Q}_{i_{k}, s}^{\mathrm{UL}}}+\mu_{j_{l}, s}^{\mathrm{UL}} \frac{\delta r_{j_{l}, s}^{\mathrm{UL}}}{\delta \mathbf{Q}_{i_{k}, s}^{\mathrm{UL}}}\right)\right|_{\overline{\mathbf{Q}}_{i_{k}, s}^{\mathrm{UL}}} .
\end{aligned}
$$

Matrices $\Upsilon_{i_{k}, s}^{\mathrm{DL}}$ in (34) and $\boldsymbol{\Upsilon}_{i_{k}, s}^{\mathrm{UL}}$ in (35) are known as interference-cost (or price) matrices (see (17)-(18)).

Therefore, using Taylor's expansion of $f_{-k}\left(\mathbf{Q}_{k, s}, \mathbf{Q}_{-k, s}\right)$ in (33) in the per-frame problem ( $\left.\mathrm{P}_{\text {frame }}^{s}\right)$ in (15) and discarding irrelevant constant terms, it is possible to approximate the perframe problem $\left(\mathrm{P}_{\text {frame }}^{s}\right)$ in (15) by a set of $K$ subproblems (one per $\mathrm{SC}$ ). The subproblem corresponding to $\mathrm{SC} k$ is the one shown in (21), as only the terms $-\operatorname{Tr}\left(\mathbf{Q}_{i_{k}, s}^{d} \mathbf{\Upsilon}_{i_{k}, s}^{d}\right)$, $\forall i_{k} \in \mathcal{I}_{k}, \forall d$ remain from (33). Note that $\overline{\mathbf{Q}}_{k, s}$ is needed to compute $\Upsilon_{i_{k}, s}^{\mathrm{DL}}, \boldsymbol{\Upsilon}_{i_{k}, s}^{\mathrm{UL}}, \forall i_{k} \in \mathcal{I}_{k}$, in (34)-(35), which can be obtained from the previous iteration.

\section{APPENDIX B \\ PROOF OF PROPOSITION 1}

Leaving the nonnegative definiteness constraint on $\mathbf{Q}_{i_{k}, s}^{d}$ implicit, the Lagrange function of problem in (22) is:

$$
\begin{aligned}
\mathcal{L}_{i_{k}, s}^{d}\left(\mathbf{Q}_{i_{k}, s}^{d}, \lambda_{i_{k}, s}^{d}\right)= & \mu_{i_{k}, s}^{d} \log _{2}\left|\mathbf{I}+\mathbf{H}_{i_{k}}^{d} \mathbf{Q}_{i_{k}, s}^{d} \mathbf{H}_{i_{k}}^{d H}\left(\mathbf{N}_{i_{k}, s}^{d}\right)^{-1}\right| \\
& -\operatorname{Tr}\left(\mathbf{Q}_{i_{k}, s}^{d} \mathbf{\Upsilon}_{i_{k}, s}^{d}\right) \\
& -\lambda_{i_{k}, s}^{d}\left(\operatorname{Tr}\left(\mathbf{Q}_{i_{k}, s}^{d}\right)-P^{\max }\right)
\end{aligned}
$$

where $\lambda_{i_{k}, s}^{d} \geq 0$ denotes the Lagrange multiplier associated to the inequality transmit power constraint in (22). The dual function for problem in (22) is given by:

$$
g\left(\lambda_{i_{k}, s}^{d}\right)=\max _{\mathbf{Q}_{i_{k}, s}^{d} \succeq \mathbf{0}} \mathcal{L}_{i_{k}, s}^{d}\left(\mathbf{Q}_{i_{k}, s}^{d}, \lambda_{i_{k}, s}^{d}\right) .
$$

Finally, the dual problem of (22) is defined as [28]:

$$
\underset{\lambda_{i_{k}, s}^{d} \geq 0}{\operatorname{minimize}} g\left(\lambda_{i_{k}, s}^{d}\right) \text {. }
$$

Since problem in (22) is convex with strictly feasible points [28], the duality gap between its optimal value and that of the dual problem in (38) is zero. Therefore, problem in (22) can be solved equivalently by solving its dual problem in (38). In order to solve the dual problem, we need to obtain the dual function $g\left(\lambda_{i_{k}, s}^{d}\right)$ in (37) for any given $\lambda_{i_{k}, s}^{d} \geq 0$. This can be done by solving the maximization problem in (37), which can 
be explicitly written (by discarding irrelevant constant terms in (36)) as:

$$
\begin{gathered}
\underset{\mathbf{Q}_{i_{k}, s}^{d} \succeq \mathbf{0}}{\operatorname{maximize}} \mu_{i_{k}, s}^{d} \log _{2}\left|\mathbf{I}+\mathbf{H}_{i_{k}}^{d} \mathbf{Q}_{i_{k}, s}^{d} \mathbf{H}_{i_{k}}^{d H}\left(\mathbf{N}_{i_{k}, s}^{d}\right)^{-1}\right| \\
-\operatorname{Tr}\left(\mathbf{B}_{i_{k}, s}^{d} \mathbf{Q}_{i_{k}, s}^{d}\right),
\end{gathered}
$$

where $\mathbf{B}_{i_{k}, s}^{d}=\boldsymbol{\Upsilon}_{i_{k}, s}^{d}+\lambda_{i_{k}, s}^{d} \mathbf{I} \in \mathbb{C}^{\tilde{M} \times \tilde{M}}$ with $\tilde{M}=M$ if $d=\mathrm{DL}$ or $\tilde{M}=N$ if $d=\mathrm{UL}$ and $\mathbf{B}_{i_{k}, s}^{d} \succeq \mathbf{0}$. To guarantee that problem (39) has a bounded objective function, $\mathbf{B}_{i_{k}, s}^{d}$ must be a full rank matrix as shown in what follows. Suppose that $\mathbf{B}_{i_{k}, s}^{d}$ is rank deficient, then we could define $\mathbf{Q}_{i_{k}, s}^{d}=q_{i_{k}, s}^{d} \mathbf{v}_{i_{k}, s}^{d} \mathbf{v}_{i_{k}, s}^{d H}$ with $q_{i_{k}, s}^{d}>0$ and $\mathbf{B}_{i_{k}, s}^{d} \mathbf{v}_{i_{k}, s}^{d}=\mathbf{0}$ such that the objective function of problem (39) results: $\mu_{i_{k}, s}^{d} \log _{2}\left|\mathbf{I}+q_{i_{k}, s}^{d} \mathbf{H}_{i_{k}}^{d} \mathbf{v}_{i_{k}, s}^{d} \mathbf{v}_{i_{k}, s}^{d H} \mathbf{H}_{i_{k}}^{d H}\left(\mathbf{N}_{i_{k}, s}^{d}\right)^{-1}\right|$, and thus by letting $q_{i_{k}, s}^{d} \rightarrow \infty$ the objective function of problem (39) goes to infinity (unbounded). Since the optimal value of problem (39) must be bounded, without loss of generality, we only need to consider the subset of $\lambda_{i_{k}, s}^{d} \geq 0$ for which $\mathbf{B}_{i_{k}, s}^{d}$ is full rank. From the KKT conditions [28] of problem (22), it follows that $\mathbf{B}_{i_{k}, s}^{d}$ is a full rank matrix when either $\lambda_{i_{k}, s}^{d}>0$ (i.e. the transmit power constraint in (22) is tight) or $\lambda_{i_{k}, s}^{d}=0$ and $\tilde{M} \leq K-1$. The last inequality has been derived from (17)-(18), as there should be at least $\tilde{M}$ summands for the matrix $\Upsilon_{i_{k}, s}^{d}$ to be full rank and we have taken into account that each SC can at most select one user in a single transmit direction such that only one term per SC from (19)-(20) does contribute to (17)-(18). Therefore, $\mathbf{B}_{i_{k}, s}^{d}$ is a full rank matrix and its inverse $\left(\mathbf{B}_{i_{k}, s}^{d}\right)^{-1}$ exists.

Similarly as in [36], define new variable $\tilde{\mathbf{Q}}_{i_{k}, s}^{d}$ as:

$$
\tilde{\mathbf{Q}}_{i_{k}, s}^{d}=\left(\mathbf{B}_{i_{k}, s}^{d}\right)^{\frac{H}{2}} \mathbf{Q}_{i_{k}, s}^{d}\left(\mathbf{B}_{i_{k}, s}^{d}\right)^{\frac{1}{2}} .
$$

Substituting (40) into problem (39) leads to:

$$
\begin{gathered}
\underset{\tilde{\mathbf{Q}}_{i_{k}, s}^{d} \succeq \mathbf{0}}{\operatorname{maximize}} \mu_{i_{k}, s}^{d} \log _{2} \mid \mathbf{I}+\mathbf{H}_{i_{k}}^{d}\left(\mathbf{B}_{i_{k}, s}^{d}\right)^{-\frac{H}{2}} \tilde{\mathbf{Q}}_{i_{k}, s}^{d}\left(\mathbf{B}_{i_{k}, s}^{d}\right)^{-\frac{1}{2}} \\
\mathbf{H}_{i_{k}}^{d H}\left(\mathbf{N}_{i_{k}, s}^{d}\right)^{-1} \mid-\operatorname{Tr}\left(\tilde{\mathbf{Q}}_{i_{k}, s}^{d}\right) .
\end{gathered}
$$

Without loss of generality, define the following eigenvalue decomposition (EVD):

$$
\begin{aligned}
& \mathbf{V}_{i_{k}, s}^{d} \mathbf{Z}_{i_{k}, s}^{d} \mathbf{V}_{i_{k}, s}^{d H}= \\
& \quad\left(\mathbf{B}_{i_{k}, s}^{d}\right)^{-\frac{1}{2}} \mathbf{H}_{i_{k}}^{d H}\left(\mathbf{N}_{i_{k}, s}^{d}\right)^{-1} \mathbf{H}_{i_{k}}^{d}\left(\mathbf{B}_{i_{k}, s}^{d}\right)^{-\frac{H}{2}},
\end{aligned}
$$

where $\quad \mathbf{V}_{i_{k}, s}^{d} \in \mathbb{C}^{\tilde{M} \times \tilde{M}} \quad$ is a unitary matrix and $\mathbf{Z}_{i_{k}, s}^{d}=\operatorname{diag}\left(z_{i_{k}, s}^{d}(1), \ldots, z_{i_{k}, s}^{d}(\tilde{M})\right) \in \mathbb{R}^{\tilde{M} \times \tilde{M}} \quad$ is a diagonal matrix. Substituting the above EVD in (42) into (41) and applying the Hadamard's inequality (e.g., see [37]), the optimal solution for $\tilde{\mathbf{Q}}_{i_{k}, s}^{d}$ in (41) is found as:

$$
\tilde{\mathbf{Q}}_{i_{k}, s}^{d}=\mathbf{V}_{i_{k}, s}^{d} \boldsymbol{\Sigma}_{i_{k}, s}^{d}\left(\mathbf{V}_{i_{k}, s}^{d}\right)^{H},
$$

being $\boldsymbol{\Sigma}_{i_{k}, s}^{d}=\operatorname{diag}\left(\sigma_{i_{k}, s}^{d}(1), \ldots, \sigma_{i_{k}, s}^{d}(\tilde{M})\right) \in \mathbb{C}^{\tilde{M} \times \tilde{M}}$ a diagonal matrix whereby each diagonal element $\sigma_{i_{k}, s}^{d}(n)$ is obtained applying the standard water-filling algorithm [37]:

$$
\sigma_{i_{k}, s}^{d}(n)=\left(\frac{\mu_{i_{k}, s}^{d}}{\ln (2)}-\frac{1}{z_{i_{k}, s}^{d}(n)}\right)^{+} .
$$

Finally, the optimal solution for $\mathbf{Q}_{i_{k}, s}^{d}$ is obtained by including (43) into (40), i.e.:

$$
\begin{aligned}
\mathbf{Q}_{i_{k}, s}^{d} & =\left(\mathbf{B}_{i_{k}, s}^{d}\right)^{-\frac{H}{2}} \tilde{\mathbf{Q}}_{i_{k}, s}^{d}\left(\mathbf{B}_{i_{k}, s}^{d}\right)^{-\frac{1}{2}} \\
& =\left(\mathbf{B}_{i_{k}, s}^{d}\right)^{-\frac{H}{2}} \mathbf{V}_{i_{k}, s}^{d} \boldsymbol{\Sigma}_{i_{k}, s}^{d}\left(\mathbf{V}_{i_{k}, s}^{d}\right)^{H}\left(\mathbf{B}_{i_{k}, s}^{d}\right)^{-\frac{1}{2}} .
\end{aligned}
$$

With the obtained dual function $g\left(\lambda_{i_{k}, s}^{d}\right)$ for any given $\lambda_{i_{k}, s}^{d}$, the dual problem (38) can be solved by searching over $\lambda_{i_{k}, s}^{d} \geq 0$ to minimize $g\left(\lambda_{i_{k}, s}^{d}\right)$. This can be done, for example, through a simple bisection method or ellipsoid method [28]. When $\lambda_{i_{k}, s}^{d}$ converges to the optimal solution for the dual problem, the corresponding $\mathbf{Q}_{i_{k}, s}^{d}$ becomes the optimal solution for problem in (22). This completes the proof.

\section{APPENDIX C \\ PROOF OF PROPOSITION 2}

If Algorithm 2 converges, it clearly converges to a limit point satisfying the set of KKT conditions of subproblems $\left(\mathrm{P}_{\mathrm{SC}}^{s, k}\right)$ in (21) (see line 8 in Algorithm 2). In addition, it can be easily shown that the set of KKT conditions of subproblems $\left(\mathrm{P}_{\mathrm{SC}}^{s, k}\right)$ in (21) constitute precisely the KKT conditions of problem $\left(\mathrm{P}_{\text {frame }}^{s}\right)$ in (15). Therefore, if convergence is achieved, the limit point will also satisfy the KKT conditions of problem $\left(\mathrm{P}_{\text {frame }}^{s}\right)$ in (15).

So, let us show that Algorithm 2 converges. Note first that the function $f_{-k}\left(\mathbf{Q}_{k, s}, \mathbf{Q}_{-k, s}\right)$ in (32) is jointly convex w.r.t. the set of matrices composing $\mathbf{Q}_{k, s}$ (i.e. $\mathbf{Q}_{i_{k}, s}^{d}, \forall i_{k} \in \mathcal{I}_{k}, d$ ), since $f_{-k}\left(\mathbf{Q}_{k, s}, \mathbf{Q}_{-k, s}\right)$ can be seen as the composition of a convex function and a linear function giving then as result a convex function [28]. Then, in the following we show that after solving problem $\left(\mathrm{P}_{\mathrm{SC}}^{s, k}\right)$ in (21) for $\mathrm{SC} k$, the objective function of problem $\left(\mathrm{P}_{\text {frame }}^{s}\right)$ in (15) is not decreased. Let $\mathbf{Q}_{k, s}^{\star \star}$ be the optimal solution to $\left(\mathrm{P}_{\mathrm{SC}}^{s, k}\right)$ in (21), i.e. $\mathbf{Q}_{k, s}^{\star \star} \triangleq\left\{\mathbf{Q}_{1_{k}, s}^{\mathrm{DL} \star \star}, \ldots, \mathbf{Q}_{I_{k}, s}^{\mathrm{DL} \star \star}, \mathbf{Q}_{1_{k}, s}^{\mathrm{UL} \star \star}, \ldots, \mathbf{Q}_{I_{k}, s}^{\mathrm{UL} \star \star}\right\}$.

Similarly as in (32), define the weighted sum-rate of the links related to $\mathrm{SC} k$ as:

$$
f_{k}\left(\mathbf{Q}_{k, s}, \mathbf{Q}_{-k, s}\right)=\sum_{i_{k} \in \mathcal{I}_{k}}\left(\mu_{i_{k}, s}^{\mathrm{DL}} r_{i_{k}, s}^{\mathrm{DL}}+\mu_{i_{k}, s}^{\mathrm{UL}} r_{i_{k}, s}^{\mathrm{UL}}\right),
$$

such that the objective function of problem $\left(\mathrm{P}_{\text {frame }}^{s}\right)$ in (15) is given by $\sum_{k \in \mathcal{K}} f_{k}\left(\mathbf{Q}_{k, s}, \mathbf{Q}_{-k, s}\right)$. Hence,

$$
\begin{aligned}
& \sum_{k \in \mathcal{K}} f_{k}\left(\mathbf{Q}_{k, s}^{\star \star}, \mathbf{Q}_{-k, s}\right)=f_{k}\left(\mathbf{Q}_{k, s}^{\star \star}, \mathbf{Q}_{-k, s}\right)+f_{-k}\left(\mathbf{Q}_{k, s}^{\star \star}, \mathbf{Q}_{-k, s}\right) \\
& \geq f_{k}\left(\mathbf{Q}_{k, s}^{\star \star}, \mathbf{Q}_{-k, s}\right)+f_{-k}\left(\overline{\mathbf{Q}}_{k, s}, \mathbf{Q}_{-k, s}\right) \\
& -\sum_{i_{k} \in \mathcal{I}_{k}}\left(\operatorname{Tr}\left(\left(\mathbf{Q}_{i_{k}, s}^{\mathrm{DL} \star \star}-\overline{\mathbf{Q}}_{i_{k}, s}^{\mathrm{DL}}\right) \mathbf{\Upsilon}_{i_{k}, s}^{\mathrm{DL}}\right)+\operatorname{Tr}\left(\left(\mathbf{Q}_{i_{k}, s}^{\mathrm{UL} \star \star}-\overline{\mathbf{Q}}_{i_{k}, s}^{\mathrm{UL}}\right) \mathbf{\Upsilon}_{i_{k}, s}^{\mathrm{UL}}\right)\right) \\
& \geq f_{k}\left(\overline{\mathbf{Q}}_{k, s}, \mathbf{Q}_{-k, s}\right)+f_{-k}\left(\overline{\mathbf{Q}}_{k, s}, \mathbf{Q}_{-k, s}\right) \\
& -\sum_{i_{k} \in \mathcal{I}_{k}}\left(\operatorname{Tr}\left(\left(\overline{\mathbf{Q}}_{i_{k}, s}^{\mathrm{DL}}-\overline{\mathbf{Q}}_{i_{k}, s}^{\mathrm{DL}}\right) \mathbf{\Upsilon}_{i_{k}, s}^{\mathrm{DL}}\right)+\operatorname{Tr}\left(\left(\overline{\mathbf{Q}}_{i_{k}, s}^{\mathrm{UL}}-\overline{\mathbf{Q}}_{i_{k}, s}^{\mathrm{UL}}\right) \mathbf{\Upsilon}_{i_{k}, s}^{\mathrm{UL}}\right)\right) \\
& =f_{k}\left(\overline{\mathbf{Q}}_{k, s}, \mathbf{Q}_{-k, s}\right)+f_{-k}\left(\overline{\mathbf{Q}}_{k, s}, \mathbf{Q}_{-k, s}\right)=\sum_{k \in \mathcal{K}} f_{k}\left(\overline{\mathbf{Q}}_{k, s}, \mathbf{Q}_{-k, s}\right),
\end{aligned}
$$


where the first inequality is a consequence of the function $f_{-k}\left(\mathbf{Q}_{k, s}, \mathbf{Q}_{-k, s}\right)$ being jointly convex w.r.t. the set of matrices composing $\mathbf{Q}_{k, s}$ and the fact that the cost matrices $\Upsilon_{i_{k}, s}^{d}$ in (17)-(18) are hermitian matrices ${ }^{5}$, and the second inequality holds since $\mathbf{Q}_{k, s}^{\star \star}$ (i.e. $\left\{\mathbf{Q}_{i_{k}, s}^{d \star \star}\right\}, \forall i_{k} \in \mathcal{I}_{k}, d$ ) is the optimal solution to problem $\left(\mathrm{P}_{\mathrm{SC}}^{s, k}\right)$ in (21). Finally, as the objective function of problem ( $\mathrm{P}_{\text {frame }}^{s}$ ) in (15) is bounded from above due to the maximum power constraints, the algorithm must converge to (at least) a local optimum.

\section{REFERENCES}

[1] N. Bhushan et al., "Network densification: the dominant theme for wireless evolution into 5G," IEEE Commun. Mag., vol. 52, no. 2, pp. 82-89, Feb. 2014.

[2] Z. Shen et al., "Dynamic uplink-downlink configuration and interference management in TD-LTE," IEEE Commun. Mag., vol. 50, no. 11, pp. 5159, Nov. 2012.

[3] 3GPP, Further advancements for E-UTRA physical layer aspects, TR 36.814, Release 9, v9.0.0, Mar. 2010.

[4] L. Wan, M. Zhou, and R. Wen, "Evolving LTE with flexible duplex," IEEE Global Commun. Conf. Workshops, pp. 49-54, Dec. 2013.

[5] 3GPP, Further enhancements to LTE time division duplex (TDD) for downlink-uplink (DL-UL) interference management and traffic adaptation, TR 36.828, Release 11, v11.0.0, Jun. 2012.

[6] D. Zhu, M. Lei, and A. Goldsmith, "Coordinated resource allocation in centralized radio access networks with dynamic downlink/uplink reconfiguration," IEEE Global Commun. Conf., pp. 3625-3630, Dec. 2013.

[7] D. Zhu and M. Lei, "Cluster-based dynamic DL/UL reconfiguration method in centralized RAN TDD with dense deployment of remote radio units," IEEE 77th Vehicular Technology Conf. (VTC Spring), pp. 1-5, Jun. 2013

[8] A. A. Dowhuszko et al., "A decentralized cooperative uplink/downlink adaptation scheme for TDD small cell networks," IEEE 24th Int. Symp. Personal Indoor and Mobile Radio Commun., pp. 1682-1687, Sep. 2013.

[9] M. S. ElBamby et al., "Dynamic uplink-downlink optimization in TDDbased small cell networks," Int. Symp. in Wireless Commun. Systems, pp. 939-944, Aug. 2014.

[10] V. Venkatasubramanian et al., "On the performance gain of flexible UL/DL TDD with centralized and decentralized resource allocation in dense 5G deployments," IEEE 25th Int. Symp. on Personal, Indoor and Mobile Radio Commun., pp. 1840-1845, Sep. 2014.

[11] A. M. Fouladgar et al., "Joint interference alignment and bi-directional scheduling for MIMO two-way multi-link networks," IEEE Int. Conf. on Commun., pp. 4126-4131, Jun. 2015.

[12] P. Mogensen et al., "Centimeter-wave concept for 5G ultra-dense small cells," IEEE 79th Vehicular Technology Conf. (VTC Spring), pp. 1-6, May 2014.

[13] Qualcomm, "The 5G unified air interface," Nov. 2015

[14] 3GPP, Small cell enhancements for E-UTRA and E-UTRAN - physical layer aspects, TR 36.872, Release 12, v12.1.0, Dec. 2013.

[15] _ Initial evaluation results of general interference avoidance and coordination for small cells, R1-131026, Samsung, May 2013.

[16] M. Peng et al., "Heterogeneous cloud radio access networks: a new perspective for enhancing spectral and energy efficiencies," IEEE Trans. Wireless Commun., vol. 21, no. 6, pp. 126-135, Dec. 2014

[17] — "System architecture and key technologies for 5G heterogeneous cloud radio access networks," IEEE Netw., vol. 29, no. 2, pp. 6-14, Mar. 2015.

[18] F. Kelly, "Charging and rate control for elastic traffic," European Trans. Telecommun., pp. 33-37, 1997.

[19] J. Lee and N. Jindal, "Dirty paper coding vs linear precoding for MIMO broadcast channels," in Asilomar Conf. Signals, Systems and Computers, Oct. 2006, pp. 779-783.

[20] X. Wang, G. B. Giannakis, and A. G. Marques, "A unified approach to QoS-guaranteed scheduling for channel-adaptive wireless networks," Proceedings of the IEEE, vol. 95, no. 12, pp. 2410-2431, Dec. 2007.

${ }^{5}$ For a differentiable convex function $f(\mathbf{A}), f(\mathbf{A}) \geq f(\mathbf{B})+$ $\operatorname{Tr}\left(\left.\left(\frac{\delta f(\mathbf{A})}{\delta \mathbf{A}}\right)^{H}\right|_{\mathbf{B}}(\mathbf{A}-\mathbf{B})\right)$ holds, where the right-hand side term corresponds to the first order Taylor expansion of $f(\mathbf{A})$ evaluated at $\mathbf{B}$.
[21] H. Kushner and G. Yin, Stochastic approximation algorithms and applications. 2nd ed. Berlin, Germany, Springer-Verlag, 2003.

[22] H. Kushner and P. A. Whiting, "Convergence of proportional-fair sharing algorithms under general conditions," IEEE Trans. on Wireless Commun., vol. 3, no. 4, pp. 1250-1259, Jul. 2004.

[23] R. Agrawal and V. Subramanian, "Optimality of certain channel-aware scheduling policies," Proc. 40th Allerton Conf. Commun., Control and Computing, pp. 636-647, Oct. 2002.

[24] C. Shi et al., "Distributed interference pricing for the MIMO interference channel," IEEE Int. Conf. Commun., pp. 1-5, Jun. 2009.

[25] A. Agustin et al., "Decentralized weighted sum rate maximization in MIMO-OFDMA femtocell networks," IEEE Global Commun. Conf., Dec. 2011.

[26] S.-J. Kim and G. B. Giannakis, "Optimal resource allocation for MIMO ad hoc cognitive radio networks," IEEE Trans. on Inf. Theory, pp. 3117 3131, May. 2011.

[27] A. Hjorungnes, Complex-calued matrix derivatives. Cambridge (UK), Cambridge University Press, 2011.

[28] S. Boyd and L. Vandenberghe, Convex optimization. Cambridge, 2004.

[29] J. Huang, R. A. Berry, and M. L. Honig, "Distributed interference compensation for wireless networks," IEEE J. Sel. Areas Commun., vol. 24, no. 5, pp. 1074-1084, May 2006.

[30] D. P. Palomar and M. Chiang, "A tutorial on decomposition methods for network utility maximization," IEEE J. Sel. Areas Commun., vol. 24, no. 8, pp. 1439-1451, Aug. 2006.

[31] C. Shi, R. A. Berry, and M. L. Honig, "Monotonic convergence of distributed interference pricing in wireless networks," in ISIT Conf. IEEE, Seoul, Korea, Jun. 2009.

[32] W. Yu, "Multiuser water-filling in the presence of crosstalk," Proc. Inf. Theory and App. Workshop, Jan. 2007.

[33] 3GPP Long Term Evolution (LTE). [Online]. Available: www.3gpp.org/.

[34] IEEE 802.16 Task Group m. [Online]. Available: www.ieee802.org/16/ $\operatorname{tgm} /$.

[35] Qualcomm, "5G waveform and multiple access techniques," Nov. 2015.

[36] R. Zhang and S. Cui, "Cooperative interference management with MISO beamforming," IEEE Trans. Signal Process., vol. 58, no. 10, pp. 54505458, Oct. 2010.

[37] T. M. Cover and J. A. Thomas, Elements of information theory. Wiley, 2006.

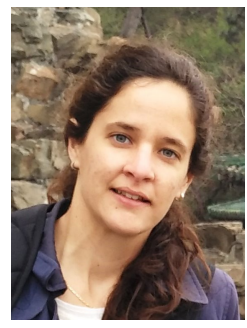

Sandra Lagen received the Telecommunications Engineering, M.S., and Ph.D. degrees from Universitat Politècnica de Catalunya (UPC), Barcelona, Spain, in 2011, 2013, and 2016, respectively. From 2010 to 2011, she was with Abertis Telecom, Barcelona. In 2012, she joined the department of Signal Theory and Communications (TSC) at UPC as a research assistant, where she has participated in the EC funded projects FREEDOM and TROPIC and worked towards her Ph.D. degree with an FPU grant from the Spanish Government. In 2015, she did a three-month research appointment at Nokia Networks and Aalborg University in Aalborg, Denmark. Her main research interests include optimization theory, interference management, MIMO, and dense networks.

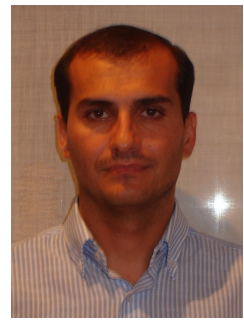

Adrian Agustin (M'08) received the M.S. degrees in Telecommunication Engineering and Electronic Engineering and the Ph.D. degree from Universitat Politècnica de Catalunya (UPC), Barcelona, Spain, in 2000, 2002 and 2008, respectively. From 2000 to 2002, he was with Indra-Espacio, Barcelona, working on research and development of code synchronization techniques for DS-CDMA. In 2002 he joined the Signal Theory and Communications department at UPC as a research assistant. In 2008, he became a research associate. He has participated in the EC funded projects SATURN, ROMANTIK, FIREWORKS, ROCKET, FREEDOM, TROPIC and TUCAN3G. His research interests include wireless multi-user MIMO, interference management and interference alignment. 


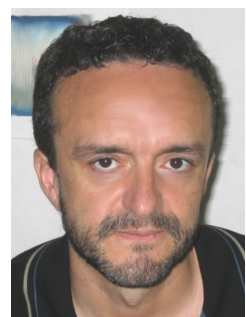

Josep Vidal (M'91) received the Telecommunication Engineering and the $\mathrm{Ph}$. D. degrees from the Universitat Politècnica de Catalunya (UPC), Barcelona, where he is Professor at the Signal Theory and Communications department. His research interests are in statistical signal processing, information and communication theory, areas in which he has authored +170 journal and conference papers. Since 2002 has coordinated collaborative EC-funded projects ROMANTIK, FIREWORKS, ROCKET, FREEDOM, TROPIC and TUCAN3G, belonging to the FP5, FP6 and FP7 programmes, all in different areas of MIMO relay communications, self-organization, cooperative transmission and heterogeneous networks. He has held research appointments with EPF Lausanne, INP Toulouse and University of Hawaii, and has organized several international workshops. He served as associate editor of IEEE Transactions on Signal Processing, and is member of the IEEE ComSoc Signal Processing for Communications and Electronics Technical Committee. 